\title{
Near-infrared polarimetric study of near-Earth object 252P/LINEAR: an implication of scattered light from the evolved dust particles
}

\author{
Yuna G. Kwon ${ }^{1}$, Masateru Ishiguro ${ }^{1}$, Jungmi Kwon ${ }^{2}$, Daisuke Kuroda ${ }^{3}$, Myungshin $\operatorname{Im}^{1}$, Changsu Choi ${ }^{1}$, \\ Motohide Tamura $^{2,4,5}$, Takahiro Nagayama ${ }^{6}$, Nobuyuki Kawai ${ }^{7}$, and Jun-Ichi Watanabe ${ }^{4}$ \\ ${ }^{1}$ Department of Physics and Astronomy, Seoul National University, 1 Gwanak, Seoul 08826, Republic of Korea \\ e-mail: ishiguro@astro.snu.ac.kr \\ 2 Department of Astronomy, Graduate School of Science, The University of Tokyo, 7-3-1 Hongo, Bunkyo-ku, \\ Tokyo 113-0033, Japan \\ ${ }^{3}$ Okayama Observatory, Kyoto University, 3037-5 Honjo, Kamogata, Asakuchi, Okayama 719-0232, Japan \\ ${ }^{4}$ National Astronomical Observatory of Japan, 2-21-1 Osawa, Mitaka, Tokyo 181-8588, Japan \\ 5 Astrobiology Center, 2-21-1 Osawa, Mitaka, Tokyo 181-8588, Japan \\ ${ }^{6}$ Faculty of Science, Kagoshima University, 21-24 Korimoto, Kagoshima, Kagoshima 890-8580, Japan \\ ${ }^{7}$ Department of Physics, Tokyo Institute of Technology, Meguro, Tokyo 152-8551, Japan
}

Received 26 March 2019 / Accepted 8 July 2019

\begin{abstract}
Context. Comets undergo resurfacing due to solar radiation, while their primordial interiors remain unchanged. Multi-epoch observations of comets enable us to characterize a change in sublimation pattern as a function of heliocentric distance, which in turn provides information on the dust environments of comets.

Aims. We aim to constrain the size and porosity of ejected dust particles from comet 252P/LINEAR and their evolution near perihelion via near-infrared (NIR) multiband polarimetry. A close approach of the comet to the Earth in March 2016 ( 0.036 au) provided a rare opportunity for the sampling of the comet at high spatial resolution.

Methods. We made NIR $J H K_{S}$-band (1.25-2.25 $\left.\mu \mathrm{m}\right)$ polarimetric observations of the comet for 12 days near perihelion, interspersed between broadband optical $(0.48-0.80 \mu \mathrm{m})$ imaging observations over four months. In addition, a dynamical simulation of the comet was performed $1000 \mathrm{yr}$ backward in time.

Results. We detected two discontinuous brightness enhancements of 252P/LINEAR. Before the first enhancement, the NIR polarization degrees of the comet were far lower than those of ordinary comets at a given phase angle. Soon after the activation, however, they increased by $\sim 13 \%$ at most, showing unusual blue polarimetric color over the $J$ and $H$ bands $\left(-2.55 \% \mu \mathrm{m}^{-1}\right.$ on average) and bluing of the dust color in both $J-H$ and $H-K_{S}$. Throughout the event, the polarization vector was marginally aligned perpendicular to the scattering plane (i.e., $\theta_{\mathrm{r}}=4.6^{\circ}-10.9^{\circ}$ ). The subsequent postperihelion reactivation of the comet lasted for approximately 1.5 months, with a factor of $\sim 30$ times pre-activation dust mass-loss rates in the $R_{C}$ band.

Conclusions. The marked increase in the polarization degree with blue NIR polarimetric color is reminiscent of the behavior of a fragmenting comet D/1999 S4 (LINEAR). The most plausible scenario for the observed polarimetric properties of 252P/LINEAR is an ejection of predominantly large (well within the geometrical optics regime) and compact dust particles from the desiccated surface layer. We conjecture that the more intense solar heating that the comet has received in the near-Earth orbit would cause the paucity of small fluffy dust particles around the nucleus of the comet.
\end{abstract}

Key words. comets: individual: 252P/LINEAR - polarization - meteorites, meteors, meteoroids

\section{Introduction}

Comets, which are among the least altered leftovers from the early solar system, have probably preserved their primitive structures inside, whereas their surfaces change from their initial states after repetitive orbital revolutions around the Sun. Solar radiation depletes near-surface volatiles and grains with high surface-to-volume ratios (Prialnik et al. 2004), while concurrent sintering effects leads to the growth of grain-to-grain contact areas and their condensation (Kossacki et al. 1994; Thomas et al. 1994; Gundlach et al. 2018). As a result, resurfacing by the inert refractory layer (called the dust mantle) makes the surface drier and more consolidated than the bulk nuclei (Biele et al. 2015; Spohn et al. 2015; Kossacki et al. 2015). The cometary dust that we observe generally originates from such a near-surface layer or bounded boulders ejected from the last apparition
(Rotundi et al. 2015; Fulle et al. 2018). Conversely, fresh particles tend to be ejected from the interiors of the nuclei in rather erratic ways, for example during a sudden brightness enhancement near perihelion or outbursts (e.g., Ishiguro et al. 2010, 2016).

The polarimetry of comets is a useful diagnostic for investigating the physical properties of dust particles, such as size and porosity (Kiselev et al. 2015). In particular, polarimetric observations at near-infrared (NIR) wavelengths $(1.2-2.3 \mu \mathrm{m})$ have been predicted to set constraints on the porosity of dust grains by covering more dust monomers (basic constitutional units of dust aggregates with $\sim 0.1 \mu$ m radii; e.g., Kimura et al. 2006) within a single wavelength than in the optical (Kolokolova \& Kimura 2010). Electromagnetic interaction between the monomers in aggregates depolarizes the light, randomizing directional information of the scattered light, so that the lower the porosity of a dust particle, the greater the depolarization as the 
wavelength increases. It is thus theoretically expected that NIR polarimetry would maximize the difference between the porosities of cometary dust, showing a more distinct contrast between the evolved hardened particles and fresh likely fluffier dust particles compared to the optical polarimetry (Kolokolova \& Kimura 2010). To date, a dozen comets have been polarimetrically observed at the NIR, with a bias toward dynamically new or long-periodic comets. Three of them were observed with multiple NIR filters (quasi-)simultaneously (i.e., comets C/1995 O1 (Hale-Bopp), 1P/Halley, and C/1975 V1 (West)); the last was the only comet observed at large phase angles of $\alpha>70^{\circ}$ (Kiselev et al. 2015 and references therein).

Here, we present the results of tracking the evolution of the activity of comet 252P/LINEAR (hereafter 252P) over four months in 2016, with multiband NIR imaging polarimetric and optical imaging observations. Comet 252P attracted attention due to its close approach to the Earth $(\sim 0.036 \mathrm{au})$ and its possible dynamical pairing with comet P/2016 BA14 (PanSTARRS). Additionally, 252P had been one of the weakly active comets (Ye et al. 2016a), despite its recent ejection into the near-Earth orbit ( $q<1.3 \mathrm{au}$, where $q$ denotes the perihelion distance), which led Ye et al. $(2016 \mathrm{a}, \mathrm{b})$ to suggest that $252 \mathrm{P}$ probably has a volatile-poor origin. However, we questioned such a scenario for the comet based on the strong $\mathrm{C}_{2}$-rich coma of $252 \mathrm{P}$ observed in 2016 (McKay et al. 2017); the observational evidence suggesting the existence of icy chunks near the nucleus of the comet (Li et al. 2017; Coulson et al. 2017); and an estimated elapsed time of 252P in the near-Earth orbit $\left(\sim 3 \times 10^{2} \mathrm{yr}\right.$; Tancredi 2014$)$, which could suffice for the growth of the dust mantle $(\sim 1-10 \mathrm{yr}$ near the Sun; Jewitt 2002; Kwon et al. 2016).

What if the long-lasting dormancy of 252P might originate in insulation by the sturdy mantle, not in a lack of volatiles? How does the light scattered by such evolved dust particles behave in comparison with the light scattered by freshly ejected particles from the interior? Our science objective is to characterize the activity of this near-Earth comet in its 2016 apparition. The favorable observational geometry of the comet in 2016 enabled us to sample the bright short-periodic comet with a high spatial resolution using ground-based telescopes.

This paper is organized as follows. In Sect. 2 we describe the observational methods and data analyses, and in Sect. 3 we present the results. Compared with the optical imaging observations conducted over $\sim$ four months, the NIR polarimetric observations only overlap for a short period of $\sim$ two weeks before the perihelion passage, and so in Sect. 3 we present the observational results of the data both taken at preperihelion, which we discuss in Sect. 4. Finally, in Sect. 5 we present a summary. The collateral postperihelion photometric results are found in Appendix A, showing the optical colorimetric results favorable for the presence of icy particles; the results of backward dynamical simulation and discussion on the size of the comet are described in Appendices B and C, respectively.

\section{Observations and data analyses}

In this section, we describe the observational methods and data analyses. The journal of our observational geometry and instrument settings is shown in Table D.1.

\subsection{Observations}

Near-infrared imaging polarimetric observations were conducted from UT 2016 March 02 to March 15 in daily cadence using a polarimeter SIRPOL of the NIR camera SIRIUS attached to the InfraRed Survey Facility (IRSF) $1.4 \mathrm{~m}$ diameter telescope at the South African Astronomical Observatory $\left(32^{\circ} 22^{\prime} 46^{\prime \prime} \mathrm{E}\right.$, $20^{\circ} 48^{\prime} 39^{\prime \prime} \mathrm{S}, 1798 \mathrm{~m}$ ), Sutherland, South Africa. SIRPOL, a single-beam polarimeter of a half-wave plate (HWP) rotator with a wire grid polarizer located upstream of the SIRIUS camera at the Cassegrain focus, simultaneously provides multiband NIR wide-field polarimetry $\left(7.7^{\prime} \times 7.7^{\prime}\right.$, with the image scale of $0.45^{\prime \prime}$ pixel $\left.^{-1}\right)$ at the $J$ (center wavelength $\left.\lambda_{C}=1.25 \mu \mathrm{m}\right), H\left(\lambda_{C}=\right.$ $1.65 \mu \mathrm{m}$ ), and $K_{S}\left(\lambda_{C}=2.25 \mu \mathrm{m}\right)$ bands (Nagayama et al. 2003; Kandori et al. 2006). A microcontroller controls the HWP rotator angle by driving a stepping motor and takes images in the sequence of $0^{\circ}, 45^{\circ}, 22.5^{\circ}$, and $67.5^{\circ}$. Because of the unavailability of the nonsidereal tracking mode, we set the exposure time to 5-20 s so that the elongation of the comet was smaller than $\sim 2^{\prime \prime}$. Target observations were conducted in units of 10 sets, each of which consists of exposures at four HWP angles $\left(0^{\circ}, 45^{\circ}\right.$, $22.5^{\circ}$, and $\left.67.5^{\circ}\right)$ dithered by $\sim 30$ pixels $\left(13.5^{\prime \prime}\right.$, which was large enough to avoid the coma signals). We interleaved sky observations +400 pixels in the $x$ direction and -400 pixels in the $y$ direction $\left( \pm 3^{\prime}\right)$ off the detector center with every 10 sets of target observations. Seeing ranged from $0.9^{\prime \prime}-1.8^{\prime \prime}$ and was primarily $1.2^{\prime \prime}$.

Preperihelion Johnson $R$-band $\left(\lambda_{C}=0.62 \mu \mathrm{m}\right)$ imaging observations were obtained from UT 2016 February 13 to March 09 using the $0.4 \mathrm{~m}$ diameter Lee Sang Gak telescope (LSGT) at the Siding Spring Observatory $\left(149^{\circ} 03^{\prime} 52^{\prime \prime} \mathrm{E}, 31^{\circ} 16^{\prime} 24^{\prime \prime} \mathrm{S}\right.$, $1165 \mathrm{~m}$ ), New South Wales, Australia. We used the SBIG ST-10 camera, whose CCD chip has $2184 \times 1472$ pixels with a $6.8 \mu \mathrm{m}$ pixel pitch. It provides an image scale of $0.48^{\prime \prime}$ with a field of view of $17.5^{\prime} \times 11.8^{\prime}$ (Im et al. 2015). Because of the unavailability of the nonsidereal tracking mode, we set the exposure time to $5-60$ s so that the elongation of the comet was smaller than $\sim 1^{\prime \prime}$. Seeing was approximately $1.2^{\prime \prime}$.

Postperihelion simultaneous multiband imaging observations with the Sloan Digital Sky Survey (SDSS) $\mathrm{g}^{\prime}$ band and the Johnson-Cousins $R_{C}$ and $I_{C}$ bands (whose $\lambda_{C}$ are 0.48 , 0.66 , and $0.80 \mu \mathrm{m}$, respectively) were performed from UT 2016 March 24 to June 10 using the $0.5 \mathrm{~m}$ diameter telescope at the Okayama Astrophysical Observatory (OAO-50; $133^{\circ} 35^{\prime} 36^{\prime \prime} \mathrm{E}$, $\left.34^{\circ} 34^{\prime} 33^{\prime \prime} \mathrm{N}, 360 \mathrm{~m}\right)$, Okayama, Japan. We employed the Multicolor Imaging Telescopes for the Survey and Monstrous Explosions (MITSuME) system, which consists of three $1024 \times 1024$ CCD chips with a $24.0 \mu \mathrm{m}$ pixel pitch (Kotani et al. 2005). It covers the field of view of $26^{\prime} \times 26^{\prime}$ with a pixel resolution of $1.53^{\prime \prime}$. Nonsidereal guiding was conducted so that we obtained integrations of 30-120 s, each depending on the signal-to-noise ratio $(\mathrm{S} / \mathrm{N})$ of the comet. Airmass was variable in the range $1.1^{\prime \prime}-5.1^{\prime \prime}$ and seeing was primarily $2.5^{\prime \prime}$.

\subsection{Data analyses}

The raw observational data (polarimetric and photometric) were preprocessed with standard techniques for imaging data: bias and dark subtractions, flat-fielding, frame registration, and sky subtraction in IRAF. The pixel coordinates of the images were converted into celestial coordinates using WCSTools (Mink 1997), with field stars listed in the Two Micron All-Sky Survey (2MASS) catalog (Skrutskie et al. 2006) for the IRSF data and with stars listed in the UCAC-3 catalog (Zacharias et al. 2010) for the LSGT and OAO data. Since incomplete sky subtraction can produce a spurious false degree of linear polarization $(P)$ signals at NIR, we took special care with the background subtraction at the level of each HWP angle image. Each set of sky frames was median-combined by matching the stars with identical WCS information and was subtracted from the target images taken at 
the same HWP angle. If there are still remaining counts after reduction by the standard sky subtraction process, we subtracted an arbitrary constant value measured at areas of blank sky well outside the coma to make the sky count nearly zero. The resulting object images were median-combined nightly by matching the instantaneous location of the comet as the center to eliminate contaminations (e.g., noise from background stars and cosmic rays) and to improve the $\mathrm{S} / \mathrm{N}$. In this process, we used data only if the $\mathrm{S} / \mathrm{N}$ of the comet in a single image is higher than three.

The $P$ of the comet was derived by performing photometry of focused images using APPHOT in IRAF. Since the $\mathrm{S} / \mathrm{N}$ of the resulting combined images $(\lessgtr 80)$ is not high enough to perform imaging polarimetry, we decided to perform aperture polarimetry. Therefore, we integrated all signals within an aperture, losing the spatial information inside. We implemented a photopolarimetric aperture of 5 pixels $\left(2.25^{\prime \prime}\right.$, corresponding to the projected physical distance of $82-173 \mathrm{~km}$ during the polarimetric observation) in radius in all bands $\left(J, H\right.$, and $\left.K_{S}\right)$. The Stokes $I$ and, subsequently, Stokes $Q$ and $U$ were calculated using

$I=\frac{\left(I_{0}+I_{45}+I_{22.5}+I_{67.5}\right)}{2}$,

and

$Q=I_{0}-I_{45}, \quad U=I_{22.5}-I_{67.5}$,

where $I_{X}$ denotes the intensity taken at a HWP angle of $X$ in degrees. The $P$ and the polarization position angle of the strongest electric vector $\theta_{\mathrm{P}}$ can be calculated using

$P=\frac{\sqrt{Q^{2}+U^{2}}}{I}$

and

$\theta_{\mathrm{P}}=\frac{1}{2} \arctan \left(\frac{U}{Q}\right)$

The above quantities ought to be corrected for instrumental effects, that is, for internal polarization, polarization efficiencies of each filter, and position angle offset of SIRPOL. The polarization efficiencies of the $J, H$, and $K_{S}$ filters are known to be $95.5 \%, 96.3 \%$, and $98.5 \%$, respectively, and the polarization position angle offset of the instrument is $105^{\circ}$ in the bands (Kandori et al. 2006). For instrumental polarization $\left(P_{\text {inst }}\right)$, as mentioned in detail in previous studies using SIRPOL (e.g., Kandori et al. 2006; Kwon et al. 2015) and in the recently updated results (Kusune et al. 2015), the $P_{\text {inst }}$ of SIRPOL is considered negligible. Since $252 \mathrm{P}$ was observed at high phase angles $\left(\alpha=78.8^{\circ}-87.0^{\circ}\right)$, where the majority of comets exhibit $P \gtrsim 20 \%$ (Kiselev et al. 2015), an influence of $P_{\text {inst }}$ on our results should be inconsequential.

Finally, the corrected $P$ and $\theta_{\mathrm{P}}$ were converted into quantities with respect to the scattering plane (the plane on which the Suncomet-Earth are located) as

$P_{\mathrm{r}}=P \cos \left(2 \theta_{\mathrm{r}}\right)$

and

$\theta_{\mathrm{r}}=\theta_{\mathrm{P}}-\left(\phi \pm \frac{\pi}{2}\right)$

where $\phi$ represents the position angle of the scattering plane, the sign of which ( \pm in Eq. (6)) was chosen to satisfy $0 \leq$ $(\phi \pm \pi / 2) \leq \pi$ (Chernova et al. 1993). Uncertainties were estimated in a standard error propagation law. We tabulated the resulting polarimetric parameters and their errors in Table 1. For polarimetric analyses, we excluded all data taken on UT 2016 March 02-03 and the data in the $K_{S}$ band on March 04 because of the faint comet brightness $(S / N \lesssim 3)$ and temporal malfunction of the $K_{S}$-band detector, respectively, and the data on March 05-08 because of high and fluctuating humidity conditions.

Photometric aperture sizes of the imaging (LSGT and OAO) and Stokes I (IRSF) data were all restricted to the projected physical distance of $1000 \mathrm{~km}$ from the center of the nucleus in radius, corresponding to 13-35 pixels for the LSGT data, 2-22 pixels for the OAO data, and 29-61 pixels for the IRSF data during the observing epochs. Sky brightness was subtracted by the circular annuli with widths of 5 pixels just outside the employed aperture. Flux calibration of the $\mathrm{g}^{\prime} R_{C} I_{C}$ filters was obtained from images of nearby comparison stars listed in the AAVSO Photometric All Sky Survey DR9 catalog (APASS; Henden et al. 2016). We assumed a systematic error of the catalog of $\sim 0.1$ mag to take into account the calibration uncertainties (Tonry et al. 2018). SDSS magnitudes of the APASS catalog were transformed to the Johnson-Cousins magnitudes using the prescriptions for the main-sequence stars by Jester et al. (2005) and Jordi et al. (2005). In addition, we converted the IRSF magnitude system, which follows the Mauna Kea Observatories NIR filter system (Tokunaga et al. 2002), into the Johnson-Cousins $R_{C}$ magnitudes to know the approximate trend of the cometary activity during the IRSF observations compared to the optical data. We analyzed the Stokes $I$ of the $H$-band data as a representative example, simply due to the highest $\mathrm{S} / \mathrm{N}$ of the comet therein. Flux calibration of the IRSF magnitudes was obtained from the field stars of the 2MASS Point Source Catalog (Curti et al. 2003) using the conversion equations of Kato et al. (2007). The $H$ magnitudes were then converted into the Johnson $R$ magnitudes, assuming a solar-like spectrum of the comet for simplicity (i.e., $R-H=$ 1.055; Binney \& Merrifield 1998), and finally converted into the $R_{C}$ magnitudes using the prescriptions of $R-R_{C}=-0.17$ (Fernie 1983). We list the apparent magnitudes of the IRSF data in Table 1.

\section{Results}

In this section, we report the results obtained by optical photometry and NIR polarimetry of comet 252P at its preperihelion.

\subsection{Photometric results I: detection of two discontinuous activations}

Figure 1 shows the temporal evolutions of (a) the absolute magnitude $m_{\mathrm{R}}(1,1,0)$, (b) $A f \rho$ parameter, and (c) dust mass-loss rate $\dot{M}_{\mathrm{d}}$ of $252 \mathrm{P}$ as a function of the Julian date (JD) during the period from UT 2016 February 13 to June 10. The arrows in panel a show the positions of two presumed points at which the comet presented discontinuous brightness enhancements. The first activation seemed to occur between March 01 and 05 (i.e., between JD 2457448.5 and 2457452.5 ), which is roughly consistent with the amateur reports by S. Yoshida ${ }^{1}$.

To investigate a change in the intrinsic brightness of the comet, it is necessary to eliminate the effect of the varying viewing geometry. Thus, we converted the apparent magnitudes into the reduced absolute magnitudes, which correspond to the magnitude at a hypothetical point in space $\left(r_{\mathrm{H}}=\Delta=1\right.$ au and $\alpha=0^{\circ}$ ), using

$m_{\mathrm{R}}(1,1,0)=m_{\mathrm{R}}\left(r_{\mathrm{H}}, \Delta, \alpha\right)-5 \log _{10}\left(r_{\mathrm{H}} \Delta\right)-2.5 \log _{10}(\Phi(\alpha))$,

1 http://www.aerith.net/comet/catalog/0252P/2016.html 
Table 1. Nightly averaged photometric and polarimetric parameters of 252P/LINEAR taken by IRSF/SIRPOL.

\begin{tabular}{|c|c|c|c|c|c|c|c|c|c|}
\hline UT 2016+ & Filter & $P^{(a)}$ & $\sigma_{\mathrm{P}}^{(b)}$ & $\theta_{\mathrm{P}}^{(c)}$ & $\sigma_{\theta_{\mathrm{P}}}^{(d)}$ & $P_{\mathrm{r}}^{(e)}$ & $\sigma_{P_{\mathrm{r}}}^{(f)}$ & $\theta_{\mathrm{r}}^{(g)}$ & $m_{\mathrm{IRSF}}{ }^{(h)}$ \\
\hline MAR 04 & $J$ & 19.46 & 0.85 & -0.68 & 1.01 & 18.12 & 1.41 & 10.69 & $13.28 \pm 0.19$ \\
\hline$\left(\phi^{(i)}=78.7^{\circ}\right)$ & $H$ & 19.57 & 0.79 & -0.46 & 0.47 & 18.17 & 0.92 & 10.91 & $12.60 \pm 0.18$ \\
\hline \multirow{3}{*}{$\begin{array}{c}\text { MAR } 09 \\
\left(\phi=82.4^{\circ}\right)\end{array}$} & $J$ & 26.04 & 0.99 & -0.34 & 0.29 & 25.19 & 0.99 & 7.33 & $12.49 \pm 0.17$ \\
\hline & $H$ & 24.00 & 0.91 & -0.38 & 0.18 & 23.23 & 0.90 & 7.28 & $11.80 \pm 0.11$ \\
\hline & $K_{S}$ & 24.05 & 0.96 & -0.24 & 0.54 & 23.25 & 1.04 & 7.43 & $11.60 \pm 0.15$ \\
\hline \multirow{3}{*}{$\begin{array}{c}\text { MAR } 11 \\
\left(\phi=83.9^{\circ}\right)\end{array}$} & $J$ & 28.19 & 1.30 & -0.43 & 0.37 & 27.61 & 1.31 & 5.79 & $12.74 \pm 0.12$ \\
\hline & $H$ & 27.88 & 1.13 & -0.37 & 0.10 & 27.31 & 1.11 & 5.85 & $12.24 \pm 0.10$ \\
\hline & $K_{S}$ & 27.74 & 1.56 & -0.15 & 1.85 & 27.12 & 2.11 & 6.10 & $12.08 \pm 0.20$ \\
\hline \multirow{3}{*}{$\begin{array}{c}\text { MAR } 12 \\
\left(\phi=84.5^{\circ}\right) \\
\end{array}$} & $J$ & 32.57 & 1.22 & 0.27 & 0.39 & 31.92 & 1.23 & 5.73 & $11.13 \pm 0.11$ \\
\hline & $H$ & 31.69 & 1.14 & 0.23 & 0.36 & 31.07 & 1.16 & 5.69 & $11.20 \pm 0.11$ \\
\hline & $K_{S}$ & 27.35 & 1.06 & 0.24 & 0.51 & 26.81 & 1.10 & 5.70 & $11.58 \pm 0.12$ \\
\hline \multirow{3}{*}{$\begin{array}{c}\text { MAR } 13 \\
\left(\phi=85.2^{\circ}\right)\end{array}$} & $J$ & 29.60 & 1.11 & 0.2 & 0.44 & 29.13 & 1.13 & 5. & $11.12 \pm$ \\
\hline & $H$ & 28.73 & 1.03 & 0.21 & 0.36 & 28.27 & 1.04 & 5.13 & $11.22 \pm 0.10$ \\
\hline & $K_{S}$ & 28.36 & 1.06 & 0.18 & 0.46 & 27.92 & 1.09 & 5.10 & $11.29 \pm 0.11$ \\
\hline \multirow{3}{*}{$\begin{array}{c}\text { MAR } 14 \\
\left(\phi=85.6^{\circ}\right)\end{array}$} & $J$ & 27.37 & 1.02 & 0.21 & 0.46 & 27.02 & 1.05 & 4.64 & $11.86 \pm 0.17$ \\
\hline & $H$ & 26.03 & 0.94 & 0.23 & 0.36 & 25.69 & 0.95 & 4.66 & $11.41 \pm 0.10$ \\
\hline & $K_{\mathrm{S}}$ & 26.19 & 0.98 & 0.19 & 0.56 & 25.85 & 1.02 & 4.62 & $11.27 \pm 0.20$ \\
\hline \multirow{3}{*}{$\begin{array}{l}\text { MAR } 15 \\
\left(\phi=85.9^{\circ}\right)\end{array}$} & $J$ & 28.10 & 1.11 & 0.19 & 0.59 & 27.73 & 1.15 & 4.68 & $11.84 \pm 0.12$ \\
\hline & $H$ & 27.28 & 1.01 & 0.26 & 0.38 & 26.91 & 1.02 & 4.75 & $11.37 \pm 0.10$ \\
\hline & $K_{S}$ & 26.87 & 1.05 & 0.29 & 0.46 & 26.49 & 1.08 & 4.77 & $11.25 \pm 0.19$ \\
\hline
\end{tabular}

Notes. ${ }^{(a)}$ Observed degree of linear polarization of the comet in percent; ${ }^{(b)}$ standard deviation of $P$ in percent; ${ }^{(c)}$ electric vector position angle in degrees; ${ }^{(d)}$ standard deviation of $\theta_{\mathrm{P}}$ in degrees; ${ }^{(e)} P$ relative to the scattering plane in percent (see Eq. $\left.(5)\right)$; ${ }^{(f)}$ standard deviation of $P_{\mathrm{r}}$ in percent; ${ }^{(g)}$ polarization angle with regard to the perpendicular direction of the scattering plane in degrees (see Eq. (6)); ${ }^{(h)}$ apparent magnitude of the IRSF data; and ${ }^{(i)}$ position angle of the scattering plane.

where $m_{\mathrm{R}}\left(r_{\mathrm{H}}, \Delta, \alpha\right)$ is the apparent magnitude of the comet and $\Phi(\alpha)$ is the phase function of the coma dust. We adopted a commonly used empirical scattering phase function $\left(2.5 \log _{10}\right.$ $(\Phi(\alpha))=b \alpha)$, where the phase coefficient of $b=0.035 \mathrm{mag} \mathrm{deg}^{-1}$ was assumed (see, e.g., Lamy et al. 2004, p. 223). Although the errors associated with the Poisson noise of the OAO data were on the order of $0.001-0.01 \mathrm{mag}$, we added the standard deviations of the field stars for the differential photometry (always $\lesssim 0.2 \mathrm{mag}$ ) and the employed photometric error of the APASS catalog $(\sim 0.1 \mathrm{mag})$ to derive the resulting uncertainties. We then estimated the $A f \rho$ parameters, a proxy of dust production rate of the comet $(A$ is the albedo of dust particles and $f$ is their packing density within the aperture radius of $\rho$; A'Hearn et al. 1984) to compare the activity level in 2016 with that of the 2000 apparition (Ye et al. 2016b) from

$A f \rho=\mathrm{Y}\left[\frac{\Delta}{\mathrm{au}}\right]^{2}\left[\frac{r_{\mathrm{H}}}{\mathrm{au}}\right]^{2}\left[\frac{\rho}{\mathrm{cm}}\right]^{-1} \times 2.512^{\left(m_{\odot}-m_{\mathrm{R}}\right)}$,

in which $m_{\odot}$ is the $R_{C}$ band magnitude of the Sun at $r_{\mathrm{H}}=1 \mathrm{au}$ $\left(m_{\odot}=-27.11\right.$; Drilling \& Landolt 2000), $Y$ is the unitary transformation factor of $8.95 \times 10^{26}$ for distances (Kwon et al. 2016), and $\rho$ is the considered aperture size $\left(10^{3} \mathrm{~km}=10^{8} \mathrm{~cm}\right.$ in this study). The resulting $A f \rho$ values range from $5.3-9.5 \mathrm{~cm}$, with an average of $7.5 \mathrm{~cm}$ before the first activation, already showing a $\sim 13$ times higher level one month prior to its perihelion passage compared to the 2000 apparition $(\sim 0.6 \mathrm{~cm}$; Ye et al. 2016b). Both the $m_{\mathrm{R}}(1,1,0)$ and $A f \rho$ values escalated sharply between March 01 and 05 by $\sim 2 \mathrm{mag}$ and by $\sim 35 \mathrm{~cm}$, respectively, implying a sudden increase in the number density of dust particles within the coma encircled by the aperture radius of $1000 \mathrm{~km}$. A lower limit of the dust mass-loss rate can be estimated from the optical photometry using an equation from Luu \& Jewitt (1992)

$$
\dot{M}_{\mathrm{d}}=\frac{1.1 \times 10^{-3} \pi \rho_{\mathrm{d}} \bar{a} \eta r_{\mathrm{obj}}^{2}}{\varrho r_{\mathrm{h}}^{1 / 2} \Delta},
$$

where $\rho_{\mathrm{d}}$ is the mass density of dust particles (nominal $1000 \mathrm{~kg} \mathrm{~m}^{-3}$ was assumed), $\bar{a}$ is the average size of small particles $(1 \mu \mathrm{m}$ was assumed; the radius of the most effective scatterers in the optical), $r_{\mathrm{obj}}$ is the radius of the comet $(300 \pm 30 \mathrm{~m}$; Li et al. 2017), $\varrho$ is the photometric aperture size in arcseconds (Table D.2), and $\eta$ is the ratio of the mean optical scattering cross-section of the coma dust $\left(C_{\mathrm{c}}\right)$ to the nucleus cross-section $\left(C_{\mathrm{n}}\right)$. We assumed a spherical nucleus with a radius of $r_{\mathrm{obj}}$ (i.e., $C_{\mathrm{n}}=\pi r_{\mathrm{obj}}^{2}$ ). The $C_{\mathrm{c}}$ can be computed in the same manner as in Luu \& Jewitt (1992)

$p_{\mathrm{R}} C_{\mathrm{c}}=2.24 \times 10^{22} \pi\left(r_{\mathrm{H}} \Delta\right)^{2} 10^{0.4\left(m_{\odot}-m_{\mathrm{R}}(1,1,0)\right)}$,

where $p_{\mathrm{R}}$ is the geometric albedo in $R_{C}$-band (0.04 was assumed). As a result, the average $\dot{M}_{\mathrm{d}}$ prior to the first activation is $0.4 \pm 0.2 \mathrm{~kg} \mathrm{~s}^{-1}$, increasing by $\sim 5.5$ times during the first activation. We note that the above estimates of $\dot{M}_{\mathrm{d}}$, assuming the size of the most efficient scatterers of $\sim 1 \mu \mathrm{m}$ in the optical wavelength, could become significantly higher if we consider large dust particles (e.g., $\bar{a}=100 \mu \mathrm{m}-1 \mathrm{~mm}$ ). Despite the simplified assumptions we employed, it would be informative to monitor a long-term variation of the activity level of the comet. We summarized our photometry in Table D.2.

The above rough estimate of the activating point from the optical photometry (between March 01-05) would be narrowed 


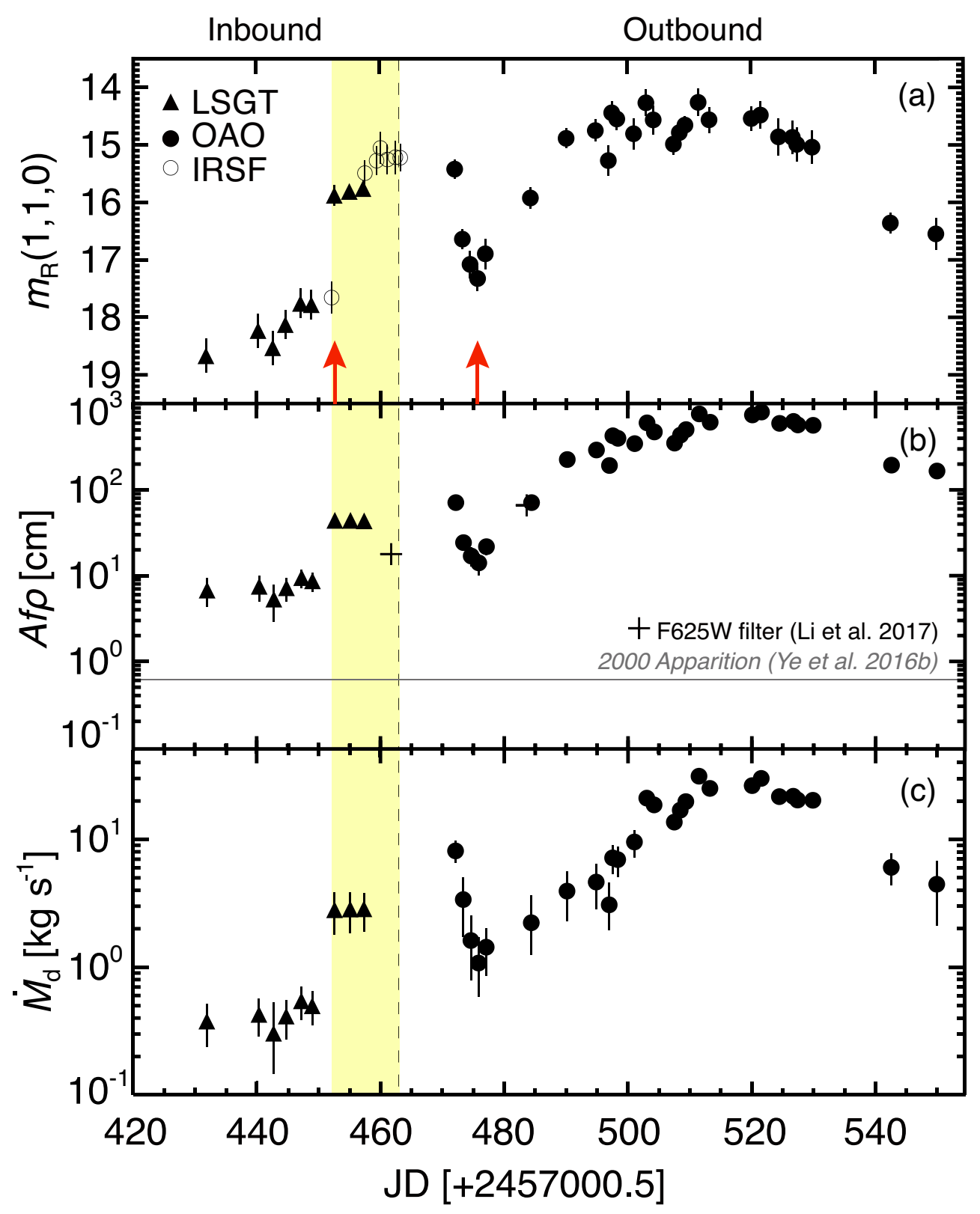

Fig. 1. (a) Absolute magnitudes $m_{\mathrm{R}}(1$, $1,0),(b) A f \rho$, and (c) dust massloss rate $\dot{M}_{\mathrm{d}}$ of $252 \mathrm{P}$ observed from UT 2016 February 13 to June 10 with LSGT/SBIG ST-10 (filled triangles), OAO-50/MITSuME (filled circles), and IRSF/SIRPOL (open circles) are shown as a function of the Julian date (JD). The dashed vertical lines denote perihelion at $r_{\mathrm{H}}=0.996 \mathrm{au}$ on UT 2016 March 15.28. The two arrows in panel a indicate the presumed locations where 252P presented significant brightness enhancements. In panel $b$ the horizontal line of $A f \rho=0.6$ denotes the median Af $\rho$ value of the comet in the 2000 apparition (Ye et al. 2016b). The two crosses are from the Hubble Space Telescope data of Li et al. (2017) observed within the aperture radius of $\lesssim 10 \mathrm{~km}$ from the center in the $\mathrm{F} 625 \mathrm{~W}$ filter. The shaded area (in yellow) covers the epoch of our polarimetric observations. using the Stokes $I$ maps of the IRSF data taken UT 2016 March 04 and 09 (open circles in Fig. 1a). Figure 2a shows azimuthally averaged surface brightness profiles of 252P from the LSGT ( $R$ band) and IRSF ( $H$ band) data on a logarithmic scale. All brightness points were normalized to the radial distance of $1.25^{\prime \prime}$. Radial gradients of the points were then compared with the slopes of $m=-1$ and $m=-1.5$, which are typical of cometary dust expanding with initial ejection velocity under the solar radiation field (Jewitt \& Meech 1987). Compared to the triangles, both decreased steadily along the $m=-1$ slope to $\sim 4^{\prime \prime}$ and along the $m=-1.5$ slope outward; the squares show flatter distributions, and in particular, the red squares on March 09 clearly show a shallower slope in the inner coma. $2 \times 2$ binned $H$-band images of the IRSF data are shown in panels (b) and (c), with the surface brightness being normalized between 0 and 1 . A development of the central whitish-yellow part before versus after the activation is evident. On March 04 a feeble coma encircled the nucleus. The dust coma on March 09, however, was enlarged, being broadly elongated in the direction of the negative velocity vector $(-v)$ with respect to the nucleus position (star symbol).
Based on (i) the sudden increases in the photometric parameters of the LSGT data between March 01 and 05 and of the IRSF data between March 04 and 09, but (ii) nearly identical and steady radial profiles of the comet on March 01 and 04, we conclude that the first brightness enhancement most likely occurred on UT 2016 March 04-05. Similarly, we present the multiband photometric results of the postperihelion reactivation of 252P that occurred on UT 2016 March 27-28 in Appendix A.

\subsection{Photometric results II: NIR dust color}

From the $I$ of the IRSF data (Eq. (1)), we obtained the colorimetric results of $252 \mathrm{P}$ dust. Figure 3 shows the temporal evolution of the $m_{\mathrm{IRSF}}$ difference of $J-H$ and $H-K_{S}$ as black and purple circles, respectively. Values of the magnitude differences calculated from $m_{\mathrm{IRSF}}$ in Table 1 are listed in Table 2. Overall, the temporal evolution in the NIR dust color of 252P appears to be in opposition to that of the comet's brightness. Before the activation (March 04.84), the $J-H$ dust color was $0.68 \pm 0.26 \mathrm{mag}$, which is consistent with the color range of cometary dust 


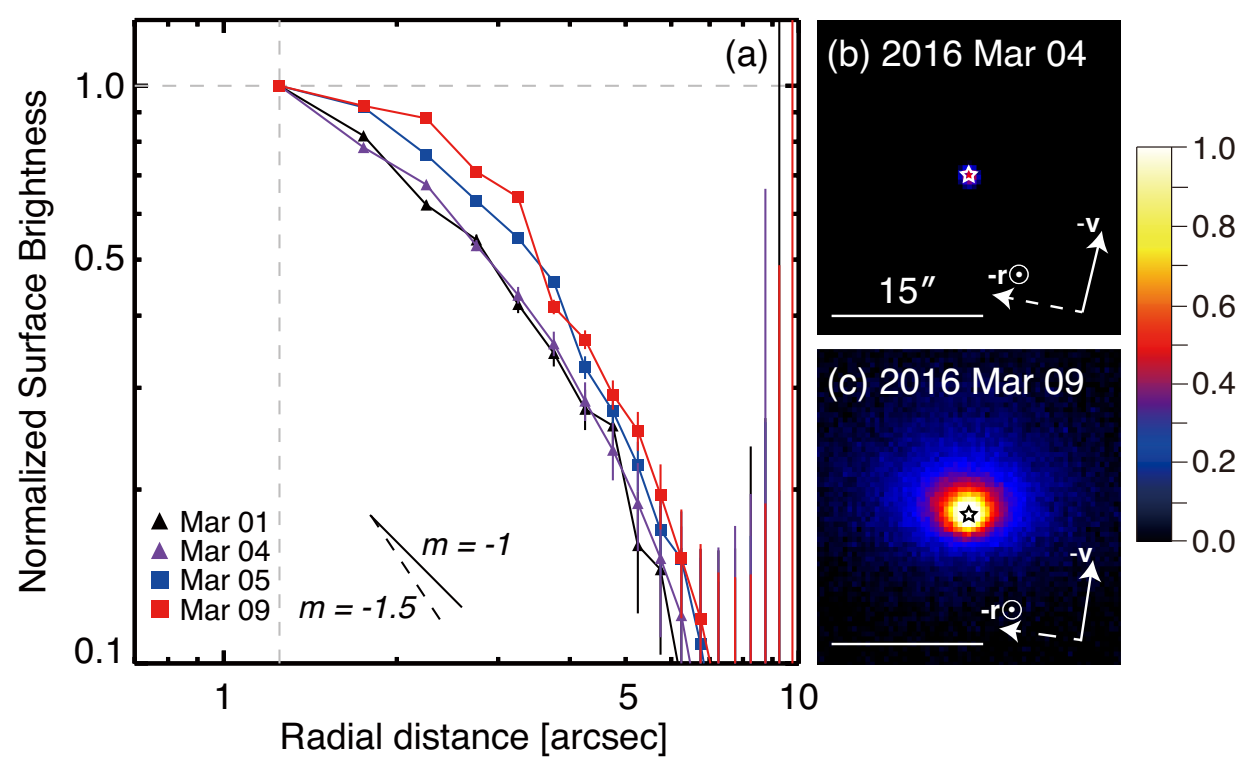

Fig. 2. Azimuthally averaged surface brightness profiles of 252P from the (a) LSGT ( $R$ band) and IRSF ( $H$ band; Stokes $I)$ data. All brightness points are normalized to the radial distance of $1.25^{\prime \prime}$. The black solid and dashed lines denote the gradients of $m=-1$ and $m=-1.5$, respectively, which are typical of cometary dust steadily expanding with initial ejection velocity under the solar radiation field (Jewitt \& Meech 1987 ). $2 \times 2$ binned $\mathrm{H}$-band images are shown, each of which are from (b) before and (c) after the first activation of 252P taken from IRSF. Brightness was normalized between 0 and 1 . North is up, and east is to the left. The bottom solid lines scale with $15^{\prime \prime}$, and the dashed and solid arrows denote the antisolar $\left(-\mathbf{r}_{\odot}\right)$ and negative velocity $(-\boldsymbol{v})$ vectors, respectively. Central stars mark the position of the nucleus.

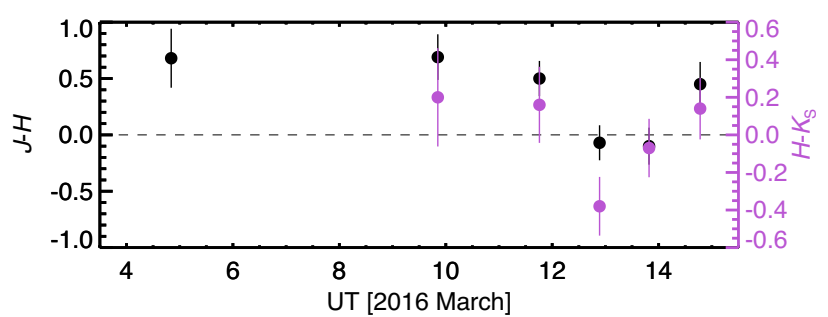

Fig. 3. Temporal evolution of the NIR color indices $\left(m_{\text {diff }}\right)$ of $252 \mathrm{P}$ dust measured from the IRSF data (Table 1). Black and purple circles denote the colors of $J-H$ and $H-K_{S}$, respectively.

measured by Jewitt \& Meech (1986). Soon after the ignition, both $J-H$ and $H-K_{S}$ values first decreased to the minima: from $0.69 \pm 0.20 \mathrm{mag}$ (March 09.85) and 0.50 $\pm 0.16 \mathrm{mag}$ (March 11.76) to $-0.07 \pm 0.16 \mathrm{mag}$ (March 12.89) and $-0.10 \pm 0.16 \mathrm{mag}$ (March 13.82) for $J-H$, and from $0.20 \pm 0.19$ mag (March 09.85) and $0.16 \pm 0.22 \mathrm{mag}$ (March 11.76) to $-0.38 \pm 0.16 \mathrm{mag}$ (March 12.89) and $-0.07 \pm 0.15 \mathrm{mag}$ (March 13.82) for $H-K_{S}$. The bluest color (i.e., minimal $m_{\mathrm{IRSF}}$ difference) occurred on March 12-13 both for $J-H$ and for $H-K_{S}$, when the comet showed the maximum brightness (Fig. 1a). Subsequently, the colors returned to the pre-activation values: $0.45 \pm 0.20 \mathrm{mag}$ (March 14.78) and $0.47 \pm 0.19 \mathrm{mag}$ (March 15.83) for $J-H$, and $0.14 \pm 0.22 \mathrm{mag}$ (March 14.78) and $0.12 \pm 0.21 \mathrm{mag}$ (March 15.83) for $H-K_{S}$.

\subsection{Polarimetric results I: phase angle dependence}

Low albedos and porous aggregate structures of cometary dust particles have led to a general dependence of $P$ with respect to the scattering plane $\left(P_{\mathrm{r}}\right)$ on the phase angle $\alpha$, parameterized by a shallow branch of negative polarization with an average minimum polarization $P_{\min } \approx-1.5 \%$ at $\alpha_{\min } \approx 10^{\circ}$, an inversion angle $\alpha_{0}$ at $\alpha \approx 22^{\circ}$, and a maximum polarization $P_{\max } \approx 25-30 \%$ at $\alpha_{\max } \approx 95^{\circ}$ in the optical and NIR (Kiselev et al. 2015).
To investigate the polarimetric behavior of 252P, we first gleaned the archival NIR polarimetric data of cometary dust from the database of comet polarimetry (DOCP; Kiselev et al. 2010, as shown in Fig. 22.3 of Kiselev et al. 2015) and later literature (Kuroda et al. 2015; Kwon et al. 2017). Fitting the average phase curve in each NIR band was obtained by employing the empirical trigonometric function of Penttilä et al. (2005),

$P_{\mathrm{r}}(\alpha)=b(\sin \alpha)^{c_{1}} \times \cos \left(\frac{\alpha}{2}\right)^{c_{2}} \times \sin \left(\alpha-\alpha_{0}\right)$,

where $b, c_{1}, c_{2}$, and $\alpha_{0}$ are the wavelength-dependent parameters for characterizing the $P_{\mathrm{r}}-\alpha$ dependence. The best fit (minimum $\chi^{2}$ ) parameters weighted by the square of the errors in the $J$, $H$, and $K\left(K_{S}\right)$ bands are described in the captions of Figs. 4-6, respectively. We consider the fitting results of the inversion angle and maximum polarization degrees to be less reliable because of the little data, or lack of data, observed at small $\left(\alpha \lesssim 25^{\circ}\right)$ and large $\left(\alpha \gtrsim 110^{\circ}\right)$ phase angle regions, despite the small fitting errors. Figures 4- 6 present $P_{\mathrm{r}}$ of comets in the $J, H$, and $K$ (and $K_{S}$ ) bands, respectively, as a function of phase angle $\alpha$. The average $\alpha$ dependences are shown as the solid curves with shaded $3 \sigma$ areas. The curves are basically the results of interpolation; thus, the error ranges of the four fitting parameters are not as large, except for the case of the $J$ band where there are no available data points at $\alpha<30^{\circ}$. In this case, we forced the curve to fit the point $(0,0)$ just for visualization.

At first glance, the $P_{\mathrm{r}}$ values of cometary dust are distributed quite homogeneously along the average phase curves, especially in the $K$ and $K_{S}$ bands, regardless of the dynamical class of comets or the observational conditions (e.g., the observing geometry). The similarity in $P_{\mathrm{r}}$ may imply that dust grains of comets have similarities to a large extent in their physical (e.g., structure and size distribution) and/or compositional properties (e.g., complex refractive index). After a careful examination, however, we found that some comets still show deviations from the average trends. In particular, long-period comet C/1995 O1 (Hale-Bopp) consistently shows higher $P_{\mathrm{r}}(\alpha)$ values than 
Table 2. Near-infrared photometric and polarimetric color indices of 252P/LINEAR from the IRSF data.

\begin{tabular}{cccccc}
\hline \hline $\begin{array}{c}\text { Median UT } \\
\text { 2016+ }\end{array}$ & $\begin{array}{c}\text { Epoch }^{(1)} \\
\text { number }\end{array}$ & $J-H^{(2)}(\mathrm{mag})$ & $H-K_{S}^{(3)}(\mathrm{mag})$ & $\mathrm{PC}_{(J-H)}{ }^{(4)}\left(\% \mu \mathrm{m}^{-1}\right)$ & $\mathrm{PC}_{\left(H-K_{S}\right)}{ }^{(5)}\left(\% \mu \mathrm{m}^{-1}\right)$ \\
\hline $03 / 0420: 16$ & 1 & $0.68 \pm 0.26$ & - & $0.13 \pm 1.68$ & - \\
$03 / 0920: 27$ & 2 & $0.69 \pm 0.20$ & $0.20 \pm 0.19$ & $-4.90 \pm 1.34$ & $0.03 \pm 1.38$ \\
$03 / 1118: 12$ & 3 & $0.56 \pm 0.16$ & $0.16 \pm 0.22$ & $-0.75 \pm 0.72$ & $-0.32 \pm 2.38$ \\
$03 / 1221: 19$ & 4 & $-0.07 \pm 0.16$ & $-0.38 \pm 0.16$ & $-2.13 \pm 1.69$ & $-7.10 \pm 1.60$ \\
$03 / 1319: 43$ & 5 & $-0.10 \pm 0.16$ & $-0.07 \pm 0.15$ & $-2.15 \pm 1.54$ & $-0.58 \pm 1.51$ \\
$03 / 1418: 49$ & 6 & $0.45 \pm 0.20$ & $0.14 \pm 0.22$ & $-3.33 \pm 1.42$ & $0.27 \pm 1.39$ \\
$03 / 1519: 56$ & 7 & $0.47 \pm 0.19$ & $0.12 \pm 0.21$ & $-2.05 \pm 1.54$ & $-0.70 \pm 1.49$
\end{tabular}

Notes. ${ }^{(1)}$ Number of the epoch described in Fig. $7 ;{ }^{(2)}$ magnitude difference measured between the $J$ and $H$ bands; ${ }^{(3)}$ magnitude difference measured between the $H$ and $K_{S}$ bands; ${ }^{(4)}$ polarimetric color of the cometary dust over the $J-H$ bands; and ${ }^{(5)}$ polarimetric color of the cometary dust over the $H-K_{S}$ bands.

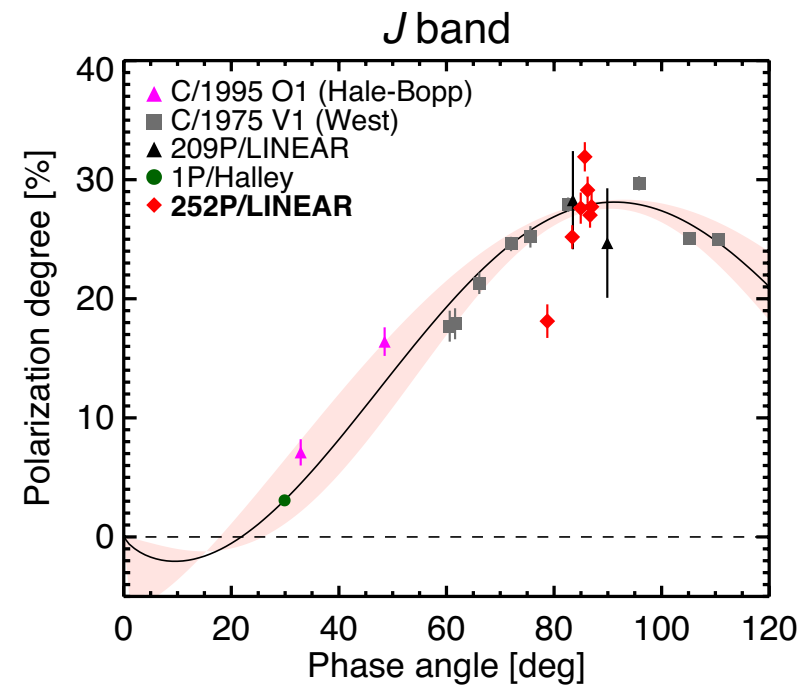

Fig. 4. $P_{\mathrm{r}}$ of comets in the $J$ band $\left(\lambda_{C}=1.25 \mu \mathrm{m}\right)$ as a function of phase angle. The data for comets C/1995 O1 (Hale-Bopp), C/1975 V1 (West), and $1 \mathrm{P} / \mathrm{Halley}$ are from the database of comet polarimetry (DOCP; Kiselev et al. 2010), and the data for comets 209P/LINEAR and C/2013 US10 (Catalina) are from Kuroda et al. (2015) and Kwon et al. (2017), respectively. The red symbols show the results for 252P. The solid curve denotes the interpolated average $\alpha$ dependence of the comets, as a result of Eq. (11). The shaded area (in pink) covers the $3 \sigma$ region of the average trend. The best fit (minimum $\chi^{2}$ ) parameters weighted by the square of the errors in $J$ band are $b=38.64 \pm 1.74 \%, c_{1}=0.77 \pm 0.31, c_{2}=$ $0.70 \pm 0.11$, and $\alpha_{0}=21.85^{\circ} \pm 2.02^{\circ}$

the majority of comets in all NIR bands (Jones \& Gehrz 2000) or even in the optical (Kwon et al. 2017), whereas short-period comets 10P/Tempel 2 and 55P/Tempel-Tuttle (Kelley et al. 2004) apparently have slightly lower $P_{\mathrm{r}}(\alpha)$ values than the average in the $K$ band.

It is noteworthy that 252P showed an abrupt change in $P_{\mathrm{r}}(\alpha)$ during our observations, especially before and soon after the sudden activation on March 04-05. In Figs. 4 and 5, $P_{\mathrm{r}}$ values on March 04 (i.e., before the first activation) were significantly lower (by $\sim 7 \%$ in the $J$ and by $\sim 5 \%$ in the $H$ bands, but no available $K_{S}$ band data due to the low $\mathrm{S} / \mathrm{N}$ of $<3$ ) than the expected $P_{\mathrm{r}}$ at given $\alpha$ (i.e., the fitted curves). Throughout the activation, however, $P_{\mathrm{r}}$ increased by $\sim 13 \%$ to the maxima on March 12-13. The $P_{\mathrm{r}}$ at perihelion $\left(\alpha \approx 87^{\circ}\right.$ on March 15.38) seems to return to normal. Such a temporal $P_{\mathrm{r}}(\alpha)$ change of the comet looks similar to the patterns of photometrically driven parameters (i.e., $m_{\mathrm{R}}$, Af $\rho$, and $\dot{M}_{\mathrm{d}}$; see Sect. 3.1).

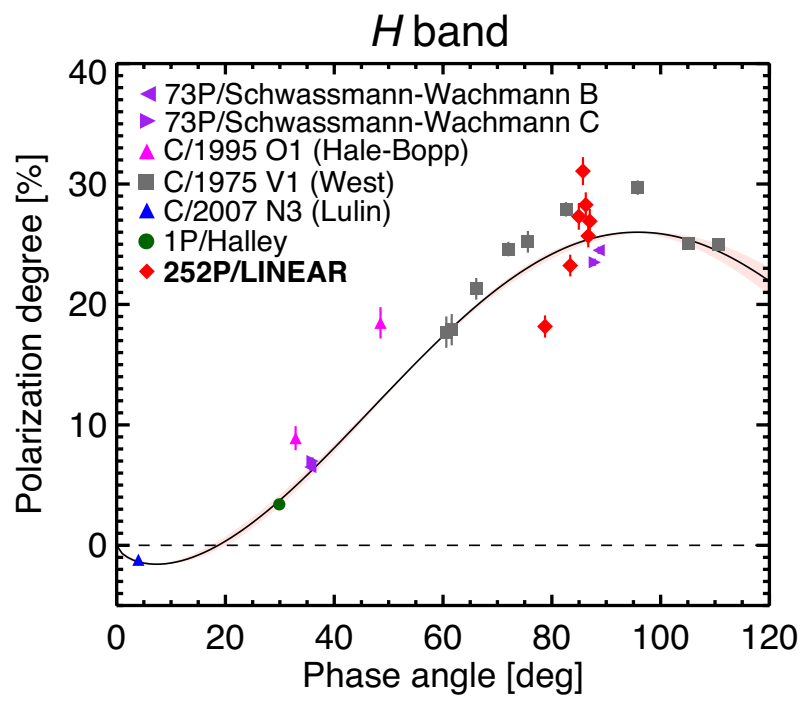

Fig. 5. Same as Fig. 4 , but in the $H$ band $\left(\lambda_{C}=1.65 \mu \mathrm{m}\right)$. All the comet data except those for 252P are from the DOCP. The best fit (minimum $\chi^{2}$ ) parameters weighted by the square of the errors in $H$ band are $b=$ $26.57 \pm 0.16 \%, c_{1}=0.63 \pm 0.04, c_{2}=(3.41 \pm 0.14) \times 10^{-9}$, and $\alpha_{0}=$ $\alpha_{0}=18.76^{\circ} \pm 0.41^{\circ}$.

The polarization vectors of the comet $\left(\theta_{\mathrm{r}}\right)$ were roughly aligned perpendicular to the scattering plane (within $4.6^{\circ}-10.9^{\circ}$ from the normal direction to the scattering plane) in the $J, H$, and $K_{S}$ bands, but presented a decreasing trend as $\alpha$ increased $\left(\theta_{\mathrm{r}}\right.$ in Table 1$)$. It should be expected that a randomly distributed scattering medium tends to signal its strongest intensity of the polarized light to the normal direction of the scattering plane (i.e., $\theta_{\mathrm{r}} \sim 0^{\circ}$; Bohren \& Huffman 1983). Breaking this condition of coma dust might lead to a nonzero value and some systematic trends of $\theta_{\mathrm{r}}$; however, we could not provide a conclusive explanation for this phenomenon from our results. It might be due to an increase in the accuracy of measurements with increasing values of the Stokes parameters. Alternatively, one of the causes might come from a substructure in the coma of 252P, indecomposable by the aperture polarimetry in this study. A series of high-resolution images taken by Hubble Space Telescope (HST) on March 14 and April 042016 suggest that 252P had a strong sunward jet, the influence of which vanished at an aperture size of more than 50-60 pixels (corresponding to $80-100 \mathrm{~km}$ in radial distance). As mentioned in Sect. 2.2, our aperture size integrated all signals within the projected radial distances of $82 \mathrm{~km}$ (on 


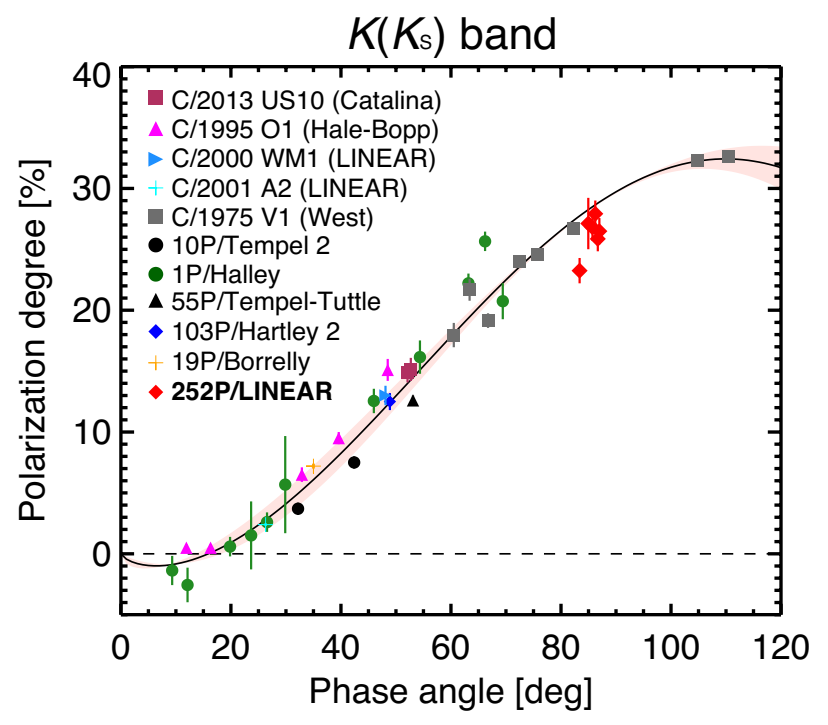

Fig. 6. Same as Fig. 4 , but in the $K$ and $K_{S}$ bands $\left(\lambda_{C}=2.20\right.$ and $2.25 \mu \mathrm{m}$, respectively). All comet data with the exception of those for C/2013 US10 (Catalina) (Kwon et al. 2017) and 252P are quoted from the DOCP. The best fit (minimum $\chi^{2}$ ) parameters weighted by the square of the errors in $K$ and $K_{S}$ bands are $b=31.47 \pm 0.18 \%, c_{1}=0.65 \pm 0.06$, $c_{2}=(6.55 \pm 0.18) \times 10^{-10}$, and $\alpha_{0}=16.97^{\circ} \pm 0.44^{\circ}$.

March 04) to $173 \mathrm{~km}$ (on March 15) during the observations, which is similar to or slightly larger than the jet scales.

\subsection{Polarimetric result II: spectral dependence}

In addition to the $\alpha$-dependence, $P_{\mathrm{r}}$ exhibits spectral dependence (called polarimetric color, $\mathrm{PC}$ ), which is defined as

$\mathrm{PC}=\frac{\Delta P_{\mathrm{r}}}{\Delta \lambda}=\frac{P_{\mathrm{r}}\left(\lambda_{2}\right)-P_{\mathrm{r}}\left(\lambda_{1}\right)}{\lambda_{2}-\lambda_{1}}$,

where $P_{\mathrm{r}}\left(\lambda_{1}\right)$ and $P_{\mathrm{r}}\left(\lambda_{2}\right)$ are the $P_{\mathrm{r}}$ values in percent measured at the center wavelengths of $\lambda_{1} \mu \mathrm{m}$ and $\lambda_{2} \mu \mathrm{m}$, respectively, and $\Delta \lambda$ is the difference between the two wavelengths $\left(\lambda_{2}-\lambda_{1}\right.$, when $\lambda_{2}>\lambda_{1}$ ) in $\mu \mathrm{m}$. Positive PC is conventionally labeled in red, and negative PC is labeled blue. PC also depends on the phase angle, but in general the PC of cometary dust is red over $\lambda=0.5-$ $1.6 \mu \mathrm{m}$ and seems to turn blue at longer wavelengths at $\alpha>25^{\circ}$ (Kolokolova et al. 2004).

Figure 7 shows the polarimetric color of 252P from the IRSF data. A sequence of the observation epochs and corresponding phase angles are shown on the left and right sides of the data points, respectively. Red on the fourth epoch (March 12) indicates the day when $252 \mathrm{P}$ showed the maximum NIR brightness during the IRSF observation (Fig. 1a). The derived values of the $\mathrm{PC}$ and their errors are listed in Table 2. Before the activation, the PC was nearly neutral (1; March 04). After the activation (2-7; March 09-15), however, the blue PC became dominant over the $J$ and $H$ bands (i.e., $-2.55 \% \mu \mathrm{m}^{-1}$ on average), while the neutral to blue PC was shown over the $H$ and $K_{S}$ bands (i.e., $-0.26 \% \mu \mathrm{m}^{-1}$ on average), except for the steep negative slope of the PC at the fourth epoch (4; red line on March 12).

The blue-dominating PC of 252P over the $J$ and $H$ bands is distinctively different from that of other comets observed at high phase angles, which showed a moderate $P_{\mathrm{r}}$ increase at this domain (e.g., $\sim 2.9 \% \mu \mathrm{m}^{-1}$ for comet $1 \mathrm{P} /$ Halley at $\alpha=65^{\circ}$ and $\sim 3.1 \% \mu \mathrm{m}^{-1}$ for comet $\mathrm{C} / 1975 \mathrm{~V} 1$ (West) at $\alpha=66^{\circ}$; Kiselev et al. 2015). Overall, 252P exhibits similar blue PC trends at NIR

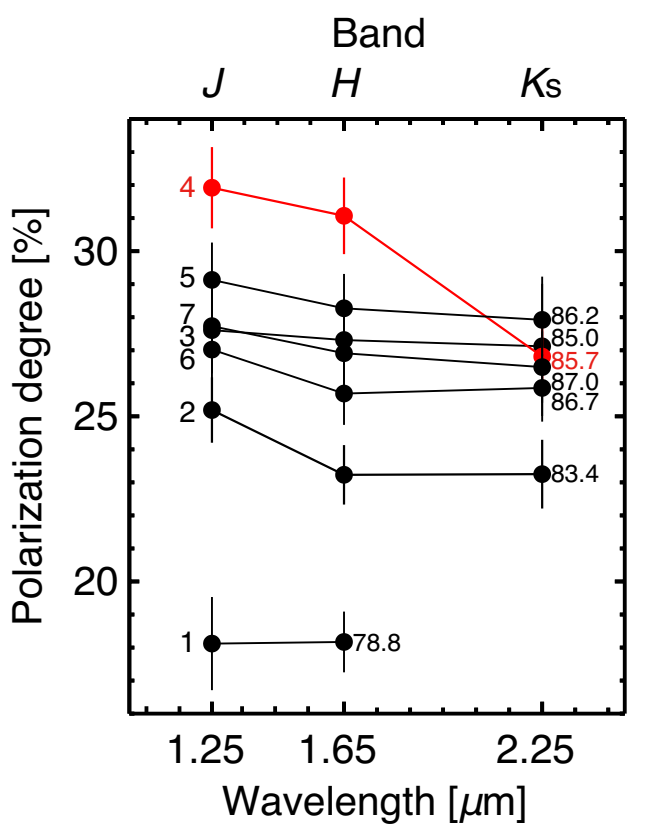

Fig. 7. $P_{\mathrm{r}}$ of $252 \mathrm{P}$ from the IRSF data (Table 1) as a function of the wavelength. The numbers on the left side of the data indicate the order of the observation epochs (1: March 04, 2: March 09, 3: March 11, 4: March 12, 5: March 13, 6: March 14, and 7: March 15), and the numbers on the right side are the phase angles of the corresponding epochs. Red on the fourth epoch (March 12) denotes the day when the comet shows the maximum NIR brightness (open circles in Fig. 1a).

during the activation, except for the steepest negative PC over the $H$ and $K_{S}$ bands at the fourth epoch (red points) when the comet exhibited the maximum brightness with the highest $P_{\mathrm{r}}$ in shorter wavelengths (open circles in Fig. 1a). Plausible scenarios to produce the observed polarimetric properties of the comet, together with the colorimetric behaviors, will be discussed in Sect. 4.1.

\section{Discussion}

In this section, we search the most likely scenario on the abrupt changes in the NIR dust color and polarimetric properties of 252P we observed and finally discuss its implication on the surface maturity of the comet.

\subsection{Abrupt change in polarization degrees near the first activation}

As shown in Sects. 3.3 and 3.4, both NIR polarization degree $P_{\mathrm{r}}$ and polarimetric color $\mathrm{PC}$ of $252 \mathrm{P}$ precipitously changed upon the first activation point only within the change in $\delta r_{\mathrm{H}}=0.008 \mathrm{au}$, which is one of the biggest and most rapid changes ever observed. It is reminiscent of comet D/1999 S4 (LINEAR), a totally disintegrated comet whose $P_{\mathrm{r}}$ in the optical increased sharply around its perihelion (Kiselev et al. 2002). By definition, $P_{\mathrm{r}}$ is the ratio of the difference of light intensities measured between perpendicular and parallel directions against the scattering plane to the total intensity. A change in $P_{\mathrm{r}}$ thus indicates a change in the physical and/or compositional properties of the dust particles responsible for light scattering as the comet approaches the Sun. Since we observed the comet with the identical instrument in simultaneous multiband imaging polarimetric modes, thereby correcting the systematic effects, it is likely that we detected the real phenomena. We considered 
three cases that could affect the polarimetric variations, together with the NIR colorimetric results (Sect. 3.2): changes in the (i) composition, (ii) effective size, and (iii) porosity of dust particles.

\subsubsection{Change in composition of dust particles}

The primary constituents of cometary dust are silicates (mainly in the form of olivine and pyroxene), carbonaceous materials (amorphous carbon and organics), Fe-bearing sulfides, and ice (Levasseur-Regourd et al. 2018). Unlike asteroids, the optical properties of which are largely differentiated with respect to heliocentric distance (DeMeo \& Carry 2014; Belskaya et al. 2017), comets seem to possess fairly homogeneous bulk optical properties with a low geometric albedo of $\sim 0.04$ (Lamy et al. 2004), showing a nearly uniform distribution on the polarimetric phase curves, regardless of dynamical classes (Kwon et al. 2017). However, any difference in a comet might be expected if we consider the grain properties with depth in the nucleus, perhaps as a result of mainly solar irradiation on the outer layers. Notably, solar heating tends to deplete near-surface volatiles (e.g., Prialnik et al. 2004), which means that the observed discontinuous brightening of 252P (Sect. 3.1) and its jet structure detected in high-resolution images ( $\mathrm{Li}$ et al. 2017) during the 2016 apparition might provide a possibility for fresh particles to be ejected from the interior of the nucleus.

Laboratory experiments and numerical modeling of comet analogs showed that changes in dust composition could make a difference to total absorptivity, which leads to a change in polarimetric properties: particles that are more transparent are subject to multiple scattering, so that the scattered light would be more depolarized. Absorptive particles, however, suppress the multiple scattering, which results in higher $P_{\mathrm{r}}$. Hence, increasing absorption with wavelength may lead to red PC, whereas decreasing absorption with wavelength may cause blue PC while leaving all other properties (i.e., size and porosity) unchanged (Gustafson \& Kolokolova 1999; Kolokolova et al. 2001, 2004; Kimura et al. 2006 and references therein).

Therefore, to satisfy the observed increase in $P_{\mathrm{r}}$ of 252P, first, the ejection of more absorptive particles is required. For carbonaceous materials, their absorption coefficients on wavelength increases in the NIR (Rouleau \& Martin 1991; Greenberg \& Li 1996; Kolokolova \& Jockers 1997), such that they produce a red PC and not our observed blue PC. On the contrary, the ejection of the compact icy or solid absorbing particles (Warren 1984, 2019), whose scattering largely depends on the single scattering of the whole particles but not as much on the absorption coefficients as fluffy ones (Kolokolova \& Jockers 1997; Gustafson \& Kolokolova 1999), allows them to contribute to the increase in $P_{\mathrm{r}}$ and probably to the blue $\mathrm{PC}$ if the size of the particles is larger than a few tens of $\mu \mathrm{m}$ (see Sect. 4.1.2 for detail discussions on the size and porosity). The idea of the ejection of compact icy particles during the discontinuous brightening around perihelion might be reconciled with the existence of sintered subsurface icy layer (Kossacki et al. 1994, 2015). Meanwhile, the bluing of the NIR color data (Sect. 3.2) could provide the additional constraint, if this is a purely compositional effect, that more transparent materials were ejected during the activation. However, the dominance of such material is incompatible with the sharp increase in $P_{\mathrm{r}}$, which indicates that factors other than the compositional effect should be the primary cause of the NIR color agent.

In summary, variations in the composition of dust particles may not be a primary factor provoking the sudden change in polarimetric parameters of the $252 \mathrm{P}$ preperihelion, but instead other properties, such as porosity and size of dust particles, should be considered as the factors responsible for the observed phenomena of the comet.

\subsubsection{Change in particle size and porosity}

Scattering regimes can be broadly classified into three types depending on the size parameter $X$ of dust $(X:=2 \pi a / \lambda$, where $a$ is the particle radius and $\lambda$ is the observation wavelength): the Rayleigh, Mie, and geometrical optics regimes in order of increasing $a$. Relatively high $P_{\mathrm{r}}(\alpha)$ can be achieved in the regimes of the Rayleigh $(X \ll 1 ; a \ll 0.2 \mu \mathrm{m}$ in the $J, a \ll 0.3 \mu \mathrm{m}$ in the $H$, and $a \ll 0.4 \mu \mathrm{m}$ in the $K_{S}$ bands) and geometrical optics $(X \gg 10 ; a \gg 2.0 \mu \mathrm{m}$ in the $J, a \gg 2.6 \mu \mathrm{m}$ in the $H$, and $a \gg$ $3.6 \mu \mathrm{m}$ in the $K_{S}$ bands).

For Rayleigh-like tiny particles in the Mie scattering regime ( $X \sim 1-10$; an order of $0.1-1 \mu \mathrm{m}$ dust in the NIR), the greater their contribution to the scattered light, the higher the observed $P_{\mathrm{r}}(\alpha)$ and the redder PC (Kolokolova et al. 2004). Unfortunately, the dominance of such particles would not explain the observed results for 252P, particularly the observed bluing of the NIR dust color (Fig. 3) and the prevailing blue PC over the $J$ and $H$ bands upon the activation (Fig. 7), and seems to conflict with the results of previous studies showing a minor role of very small particles in mass density (i.e., dynamics) (Fulle et al. 2015, 2016; Rotundi et al. 2015), and in light scattering of cometary dust (Jewitt \& Meech 1986; Kolokolova et al. 2007). Therefore, we dismiss the possibility that the observed polarimetric changes were largely stimulated by the increase in Rayleigh (or Rayleigh-like) particles, although not ruling out their possible existence evidenced by the change in the optical brightness (Sect. 3.1) and observed jet-like structure in the optical (Li et al. 2017). Meanwhile, such small dust particles may thermally depolarize the $K_{S}$-band data (e.g., blue PC over the $H$ and $K_{S}$ bands in Fig. 7), but we preclude the predominance of such a possibility based on the $P_{\mathrm{r}}(\alpha)$ of 252P in the $K_{S}$ band near perihelion having similar values to the points of comet C/1975 V1 (West) (gray squares; Oishi et al. 1978) from which thermal flux was subtracted and based on the case of C/1975 V1 (West), whose thermal depolarization effect was negligible at $r_{\mathrm{H}} \gtrsim 0.9$ au (Oishi et al. 1978).

Large dust particles in the geometrical optics regime ( $a>$ a few tens of $\mu \mathrm{m}$ ) can be further considered with two different porosities: fluffy (porous) and compact particles, which are in fact the two main populations of dust particles of comet 67P/Churyumov-Gerasimenko (67P) collected by Rosetta/GIADA (Fulle et al. 2015). In the case of large fluffy particles - with tensile strength of $<10^{5} \mathrm{~N} \mathrm{~m}^{-2}$ (Mendis 1991) and high charge-to-mass ratio (Fulle et al. 2015) - they behave similarly to individual constituent particles in dynamics (Mukai et al. 1992), in light scattering (Kolokolova 2011), and in thermal emissions (Wooden 2002; Kolokolova et al. 2007). Accordingly, the polarimetric properties of scattered light by such large fluffy dust particles would be similar to those by particles approaching the Rayleigh regime (but are larger than $X=1$ ) (i.e., an increase in $P_{\mathrm{r}}$ with the red $\mathrm{PC}$ ), which again contradicts the observed results of 252P.

Finally, we consider to what extent large compact dust particles contributed to the observed polarimetric properties of 252P. A dominance of these particles was corroborated by the results of Rosetta/GIADA, showing that millimeter- to centimeter-sized compact chunks of $67 \mathrm{P}$ ejected near perihelion, along with the release of the upper desiccated layer with a higher refractory-toice mass ratio than that inside the nucleus, accounted for $>85 \%$ 
of the dust mass-loss and luminosity function of the comet (e.g., Blum et al. 2017; Fulle et al. 2018). As the interaction energy between ambient dipoles (i.e., monomers) are inversely proportional to the cube of the distance between the two (Jackson 1962), less porous materials are more subject to the scattered light from neighboring dust constituents. Accordingly, as the wavelength of the incident light increases (i.e., more monomers are covered in a single wavelength), the $P_{\mathrm{r}}$ of such compact particles decreases more rapidly than that of the porous particles due to the enhanced electromagnetic interaction (Gustafson \& Kolokolova 1999; Kolokolova \& Kimura 2010). Compared to the nonperiodic and long-period comets observed at similar phase angles, it is more likely that the near-surface of $252 \mathrm{P}$ has a paucity of small fluffy dust particles owing to its more frequent perihelion passages in the near-Earth orbit (e.g., Li \& Greenberg 1998; Kolokolova et al. 2007). The circumstance would emerge as the unusual blue PC of the comet. For this reason the dominance of signals by the ejection of large compact particles into the coma would be attributable to both a sudden increase in $P_{\mathrm{r}}$ and the enhanced blue PC upon the first activation of 252P.

\subsubsection{Tentative conclusions on particle properties ejected during the activation}

The ejection of particles that are large (i.e., $a$ is at least greater than a few tens of $\mu \mathrm{m}$ in the geometrical optics regime) and compact (likely as low as the porosity of $\sim 30-65 \%$ of the nearsurface Rosetta/Philae landing site of 67P; Spohn et al. 2015) can also be deduced from the morphology of the dust coma and the color change of the apparent magnitudes of the IRSF data. Figure $2 \mathrm{c}$ shows a broad extension of the dust cloud to the trailing direction $(-v)$ with respect to the photocenter, alluding to the ejection of dust particles that are insensitive to the solar radiation pressure at $r_{\mathrm{H}} \lesssim 1 \mathrm{au}$. This means that the primary factor that distributes the ejected particles in the $-\boldsymbol{v}$ direction should instead be an explosive mechanism, such as a rocket force from sublimating gas with nonzero ejection velocity (Kelley et al. 2013), which is most likely responsible for the discontinuous brightening of the comet. Concurrently, the $J-H$ and $H-K_{\mathrm{S}}$ colors of the comet decreased with time (Fig. 3) from the typical color of cometary dust before the activation (e.g., Jewitt \& Meech 1986) to the blue-neutral color. It may also support our scenario that the dust particles that are brighter at the longer wavelengths contributed more to the intensity of the comet than the smaller or large porous dust particles. Taken as a whole, the above conjectures may answer our first question: the evolved near-surface dust layer would primarily define the activity of the comet as long as the incoming solar heat flux is sufficiently large.

\subsection{Implication for evolved surface}

Our second question is how useful NIR polarimetry would be to discriminate the behaviors of fresh and evolved dust particles. The discussion on the observed polarimetric properties largely induced by the evolved dust particles of $252 \mathrm{P}$ in Sect. 4.1 could advocate for the potential usefulness of this approach.

Returning to Fig. 6, the $P_{\mathrm{r}}(\alpha)$ of 11 comets in the $K$ band, where the number of observed comets is to date the highest among the NIR bands, follows the average trend quite well, while a careful check reveals that nonperiodic or long-period comets tend to show, on average, higher $P_{\mathrm{r}}(\alpha)$ values, particularly than two Jupiter-family comets (JFCs), 10P/Tempel 2 and 55P/Tempel-Tuttle (Kelley et al. 2004). Interestingly, the two are characterized by weak to absent $10 \mu \mathrm{m}$ silicate emission features and a slight temperature excess for the blackbody temperature (Lynch et al. 1995, 2000), both suggesting a lack of small (micrometer-sized) and/or fluffy dust particles (Hanner 1999; Wooden 2002; Lisse et al. 2002). Given that JFCs orbit closer to the Sun (which offers a more favorable environment to develop the consolidated dust mantles on their surfaces) than nonperiodic or long-period comets, it would be understandable for JFCs to show lower $P_{\mathrm{r}}(\alpha)$ at NIR than those of less-heated comets. The orbital evolution of 252P in the near-Earth orbit over $300 \mathrm{yr}$ or even more would be in favor of this scenario (Appendix B). However, for the moment, we should be cautious before drawing any firm conclusions. A single $P_{\mathrm{r}}(\alpha)$ point of a comet does not provide much information on its physical and compositional properties, but rather, we need (quasi-)simultaneous multiband polarimetric data to measure the PC of dust particles for estimation of the porosity of dust and/or comets observed at multiple observation epochs, including the high- $\alpha$ region, to trace the variation of the $P_{\mathrm{r}}(\alpha)$ and PC. Further NIR polarimetric observations undertaken in a well-organized manner are highly desirable in order to investigate secular evolutions of polarimetric parameters of cometary dust on the orbital motion and to search for any systematic differences in them between different dynamical groups of comets.

\section{Summary}

We presented multiband NIR imaging polarimetric observations around the perihelion passage and optical imaging observations of comet 252P/LINEAR taken over four months in its 2016 apparition. We summarize the main results as follows:

1. We detected two discontinuous brightness enhancements of 252P: one in the inbound orbit (on UT 2016 March 04-05) and the other in the outbound orbit (March 27-28). A month prior to the perihelion passage, $252 \mathrm{P}$ already showed $\sim 13$ times higher $A f \rho$ values than those of the 2000 apparition.

2. Upon the first activation, $m_{\mathrm{R}}(1,1,0)$ and $A f \rho$ of $252 \mathrm{P}$ derived from the optical $R$ and $R_{C}$ bands data increased by $\sim 2$ mag and $\sim 35 \mathrm{~cm}$, respectively. The attendant $\dot{M}_{\mathrm{d}}$ for the assumed $1 \mu \mathrm{m}$ particles increased to $\sim 5.5$ times the initial state. In the meantime, the $J-H$ and $H-K_{S}$ dust color values of 252P had both decreased, showing the bluest color in the middle of the activation.

3. Before the first activation, the $P_{\mathrm{r}}$ values of $252 \mathrm{P}$ were far lower than the average trend of comets at given $\alpha$, by $\sim 7$ and $\sim 5 \%$ in the $J$ and $H$ bands, respectively. Upon and soon after the activation, however, the $P_{\mathrm{r}}$ increased in all NIR bands by $\sim 13 \%$ at most, showing the distinctive development of the blue PC over $1.25-2.25 \mu \mathrm{m}$ similar to but stronger than the behaviors of a fragmenting comet D/1999 S4 (LINEAR) (Kiselev et al. 2002). In particular, the blue-dominating PC at the $J-H$ bands $\left(-2.55 \% \mu \mathrm{m}^{-1}\right.$ on average) is different from other comets observed, which show moderately red PC values at similar phase angles.

4. The most likely implication of the sudden change in the observed polarimetric properties of the comet during the activation (i.e., increased $P_{\mathrm{r}}$ with the blue PC) and the bluing of the NIR dust color would be the dominance of large (i.e., well located in the geometrical optics regime at NIR) compact particles predominantly ejected from the desiccated dust layer. The paucity of small fluffy dust particles around the nucleus of $252 \mathrm{P}$ would be ascribed to the more intense solar heating effects the comet has received in the near-Earth orbit (Appendix B) than for the less-heated nonperiodic or long-period comets observed to date. 
Acknowledgements. We thank the referee, L. Kolokolova, whose careful reading and constructive comments improved our manuscript. Y.G.K. was supported by the Global Ph.D. Fellowship Program through a National Research Foundation of Korea (NRF) grant funded by the Ministry of Education (NRF2015H1A2A1034260). This work at Seoul National University was also supported by the NRF funded by the Korean Government (MEST) grant No 2018R1D1A1A09084105. J.K. was supported by Astrobiology Center of NINS. MI and CC acknowledge the support from the National Research Foundation of Korea (NRF) grant No. 2017R1A3A3001362. M.T. was supported by MEXT/JSPS KAKENHI grant Nos. 18H05442, 15H02063, and 22000005. The IRSF project is a collaboration between Nagoya University and the South African Astronomical Observatory (SAAO) supported by Grants-in-Aid for Scientific Research on Priority Areas (A; No. 10147207 and No. 10147214) and Optical \& Near-Infrared Astronomy Inter-University Cooperation Program, from the Ministry of Education, Culture, Sports, Science and Technology (MEXT) of Japan and the National Research Foundation (NRF) of South Africa.

\section{References}

A'Hearn, M. F., Schleicher, D. G., Millis, R. L., Feldman, P. D., \& Thompson, D. T. 1984, AJ, 89, 579

Barnes, T. F., A'Hearn, M. F., \& Kolokolova, L., (Eds.), Properties of Comet Nuclei (PDS4 Format), urn:nasa:pds:compil-comet:nuc_properties::1.0, NASA Planetary Data System, 2019

Belskaya, I. N., Fornaxier, S., Tozzi, G. P., et al. 2017, Icarus, 284, 30

Biele, J., Ulamec, S., Maibaum, M., et al. 2015, Science, 349, aaa9816

Binney, J., \& Merrifield, M. 1998, Galactic Astronomy (Princeton, NJ: Princeton University Press)

Blum, J., Gundlach, B., Krause, M., et al. 2017, MNRAS, 469, 755

Bohren, C., \& Huffman, D. 1983, Absorption and Scattering of Light by Small Particles (New York: Wiley Sons)

Bonev, T., Boehnhardt, H., \& Borisov, G. 2008, A\&A, 480, 277

Chambers, J. E. 1999, in Impact of Modern Dynamics in Astronomy, eds. J., Henrard, \& S., Ferraz-Mello (Berlin: Springer), 449

Chernova, G. P., Kiselev, N. N., \& Jockers, K. 1993, Icarus, 103, 144

Coulson I., M., Cordiner, M. A., Kuan, Y.-J., et al. 2017, AJ, 153, 169

Curti, R. M., Skrutskie, M. F., van Dyk, S., et al. 2003, VizieR On-line Data Catalog: II/246

DeMeo, F. E., \& Carry, B. 2014, Nature, 505, 629

Drilling, J. S., \& Landolt, A. U. 2000, in Astrophysical Quantities 4th edn., ed. A. N. Cox (New York: Springer), 381

Fernández, J. A., Tancredi, G., Rickman, H., \& Licandro, J. 1999, A\&A, 352, 327

Fernie, J. D. 1983. PASP, 95, 782

Fulle, M., Corte, D., Rotundi, V., et al. 2015, ApJL, 802, 12

Fulle, M., Altobelli, N., Buratti, B., et al. 2016, MNRAS, 462, 2

Fulle, M., Blum, J., Green, S. F., et al. 2018, MNRAS, 482, 3326

Greenberg, J. M., \& Li, A. 1996, A\&A, 309, 258

Greenstreet, S., Ngo, H., \& Gladman, B. 2012, Icarus, 217, 355

Gundlach, B., Ratte, J., Blum, J., Oesert, J., \& Gorb, S. N. 2018, MNRAS, 479, 5272

Gustafson, B. Å. S., \& Kolokolova, L. 1999, J. Geophys. Res., 104, 31711

Hanner, M. S. 1999, Space Sci. Rev., 90, 99

Henden, A. A., Templeton, M., Terrell, D., et al. 2016, VizieR Online Data Catalog: II/336

Im, M., Choi, C., \& Kim, K. 2015, J. Korean Astron. Soc., 48, 207

Ishiguro, M., Watanabe, J.-I., Sarugaku, Y., et al. 2010, ApJ, 714, 1324

Ishiguro, M., Kuroda, D., Hanayama, H., et al. 2016, AJ, 152, 169

Jackson, J. D. 1962, Classical Electrodynamics (Hoboken, NJ: John Willey \& Sons), 641

Jester, S., Schneider, D. P., Richards, G. T., et al. 2005, AJ, 130, 873

Jewitt, D. 2002, AJ, 123, 1039

Jewitt, D. 2004, Comets II (Tucson: University of Arizona Press), 659

Jewitt, D., \& Meech, K. 1986, ApJ, 310, 937

Jewitt, D., \& Meech, K. 1987, ApJ, 317, 992

Jones, T. J., \& Gehrz, R. D. 2000, Icarus, 143, 338

Jordi, K., Grebel, E. K., \& Ammon, K. 2005, A\&A, 460, 339

Kandori, R., Kusakabe, N., Tamura, M., et al. 2006, Proc. SPIE, 6269, 626951

Kato, D., Nagashima, C., Nagayama, T., et al. 2007, PASJ, 59, 615

Keller, H. U., Mottola, S., Hviid, S. F., et al. 2017, MNRAS, 469, 357

Kelley, M. S., Woodward, C. E., Jones, T. J., Reach, W. T., \& Johnson, J. 2004 AJ, 127, 2398

Kelley, M. S., Lindler, D. J., Bodewits, D., et al. 2013, Icarus, 222, 634

Kimura, H., Kolokolova, L., \& Mann, I. 2006, A\&A, 449, 1243

Kiselev, N., Jockers, K., \& Rosenbush, V. 2002, Earth Moon Planet, 90, 167

Kiselev, N., Velichko, S., Jockers, K., Rosenbush, V., \& Kikuchi, S. 2010, NASA Planetary Data System, Database of Comet Polarimetry (DOCP), EAR-C-COMPIL-5-COMET-POLARIMETRY-V1.0, 8124
Kiselev, N., Rosenbush, V., Kolokolova, L., \& Levasseur-Regourd, A.-Ch. 2015, in Polarimetry of Stars and Planetary Systems, eds. L. Kolokolova, J. Hough, \& A. Levasseur-Regourd (Cambridge: Cambridge University Press), 379

Kolokolova, L. 2011, ASPC, 449, 345

Kolokolova, L., \& Jockers, K. 1997, Planet. Space Sci., 45, 1543

Kolokolova, L., \& Kimura, H. 2010, A\&A, 513, A40

Kolokolova, L., Jockers, K., Gustafson, B. Å. S., \& Lichtenberg, G. 2001, J. Geophys. Res., 106, 10113

Kolokolova, L., Hanner, M. S., Levasseur-Regourd, A.-C., \& Gustafson, B. Å. S. 2004, in Comets II, eds. M. Festou, H. U. Keller, \& H. A. Weaver (Tucson: University of Arizona Press), 577

Kolokolova, L., Kimura, H., Kiselev, N., \& Rosenbush, V. 2007, A\&A, 463, 1189

Kossacki, K. J., Kömle, N. I., Kargl, G., \& Steiner, G. 1994, Planet. Space Sci., 42, 393

Kossacki, K. J., Spohn, T., Hagermann, A., Kaufmann, E., \& Kührt, E. 2015, Icarus, 260, 464

Kotani, T., Kawai, N., Yanagisawa, K., et al. 2005, Nuovo Cimento C, 28, 755

Kuroda, D., Ishiguro, M., Watanabe, M., et al. 2015, ApJ, 814, 156

Kusune, T., Sugitani, K., Miao, J., et al. 2015, ApJ, 798, 60

Kwon, J., Tamura, M., Hough, J., et al. 2015, ApJ, 824, 85

Kwon, Y. G., Ishiguro, M., Hanayama, H., et al. 2016, ApJ, 818, 67

Kwon, Y. G., Ishiguro, M., Kuroda, D., et al. 2017, AJ, 154, 173

Lamy, P. L., Toth, I., Fernandez, Y. R., \& Weaver, H. A. 2004, in Comets II, eds M. C. Festou, et al. (Tuczon: University of Arizona Press), 223

Levasseur-Regourd, A. C., Jessica, A., Hervé, C., et al. 2018, Space Sci. Rev., 214, 64

Li, A., \& Greenberg, J. M. 1998, A\&A, 338, 364

Li, J. Y., Kelley, M. S., Samarasinha, N. H., et al. 2017, AJ, 154, 136

Lisse, C. M., A’Hearn, M. F., Fernández, Y. R., \& Peschke, S. B. 2002, in Dust in the Solar System and Other Planetary Systems, A Search for Trends in Cometary Dust Emission, IAU Coll. 181, eds. S. F. Green, I. P. Williams, J. A. M. McDonnell, \& N. McBride (Canterbury: ASP), 259

Luu, J. X., \& Jewitt, D. C. 1992, AJ, 104, 2243

Lynch, D. K., Hackwell, J. A., Edelsohn, D., et al. 1995, Icarus, 114, 197

Lynch, D. K., Russell, R. W., \& Sitko, M. L. 2000, Icarus, 144, 187

McKay, A. J., Kelley, M. S. P., Bodewits, D., et al. 2017, Asteroids, Comets, Meteors, 3b4, http://acm2017.uy/abstracts/Parallel3.b.4.pdf

Meech, K. J., \& Svoreň J. 2004, in Comets II, ed. M. Festou, H. U. Keller, \& H. A. Weaver (Tucson: University of Arizona Press), 317

Mendis, D. A. 1991, Astrophys. Space Sci., 176, 163

Mink, D. J. 1997, in Astronomical data Analysis Software and Systems VI, eds. G. Hunt, \& H. E. Payne, ASP Conf. Ser. 125, 249

Morbidelli, A., Bottke, Jr. W. F., Froeschlé, C., \& Michel, P. 2002, in Asteroids III, eds. W. F. Bottke, Jr. et al. (Tucson: University of Arizona Press), 409

Mukai, T., Ishimoto, H., Kozasa, T., Blum, J., \& Greenberg, J. M. 1992, A\&A, 262,315

Nagayama, T., Nagashima, C., Nakajima, Y., et al. 2003, Proc. SPIE, 4841, 459

Naidu, S. P., Benner, L. A. M., Brozovic, M., et al. 2016, in AAS/DPS Meeting 48 Abstract, 219.05

Ohtsuka, K., Sekiguchi, T., Kinoshita, D., et al. 2006, A\&A, 450, L25

Oishi, M., Kawara, K., Kobayashi, Y., et al. 1978, PASJ, 30, 149

Penttilä, A., Lumme, K., Hadamcik, E., \& Levasseur-Regourd, A.-C. 2005, A\&A, 432,1081

Perovich, D. K. 1998, J. Geophys. Res., 103, 5563

Prialnik, D., Benkhoff, J., \& Podolak, M. 2004, Comets II (Tucson: University of Arizona Press), 359

Rickman, H., Fernandez, J. A., \& Gustafson, B. A. S. 1990, A\&A, 237, 524

Reddy, V. 2016, NASA/JPL News, Comet Flying by Earth Observed with Radar and Infrared, 2016 March 24

Rotundi, A., Sierks, H., Della, C., et al. 2015, Science, 347, aaa3905

Rouleau, F., \& Martin, P. G. 1991, ApJ, 377, 526

Rudawska, R., Vaubaillon, J., Tóth, J., \& Raetz, S. 2016, in AAS/DPS Meeting 48 Abstract, 218.01

Skrutskie, M. F., Cutri, R. M., Stiening, R., et al. 2006, AJ, 131, 1163

Solontoi, M., Ivezić, Ž., Jurić, M., et al. 2012, Icarus, 218, 571

Southworth, R. B., \& Hawkins, G. S. 1963, Smithsonian Contributions to Astrophysics (Washington D.C.: Smithsonian Institution), 7, 261

Spohn, T., Knollenber, J., Ball, A. J., et al. 2015, Science, 349, aab0464

Tancredi, G. 2014, Icarus, 234, 66

Tokunaga, A. T., Simons, D. A., \& Vacca, W. D. 2002, PASP, 114, 180

Tonry, J. L., Denneau, L., Flewelling, H., et al. 2018, ApJ, 867, 105

Thomas, H., Ratke, L., \& Kochan, H. 1994, Adv. Space Res. 12, 207

Warren, S. G. 1984, Appl. Opt. 23, 1206

Warren, S. G. 2019, Phil. Trans. R. Soc. A, 377, 20180161

Wooden, D. 2002, Earth Moon Planet, 89, 247

Ye, Q.-Z., Hui, M.-T., Brown, P. G., et al. 2016a, Icarus, 264, 48

Ye, Q.-Z., Brown, P. G., \& Wiegert, P. A. 2016b, ApJ, 818, L29

Zacharias, N., Finch, C., Girard, T., et al. 2010, AJ, 139, 2184 
Appendix A: Postperihelion activation of 252P
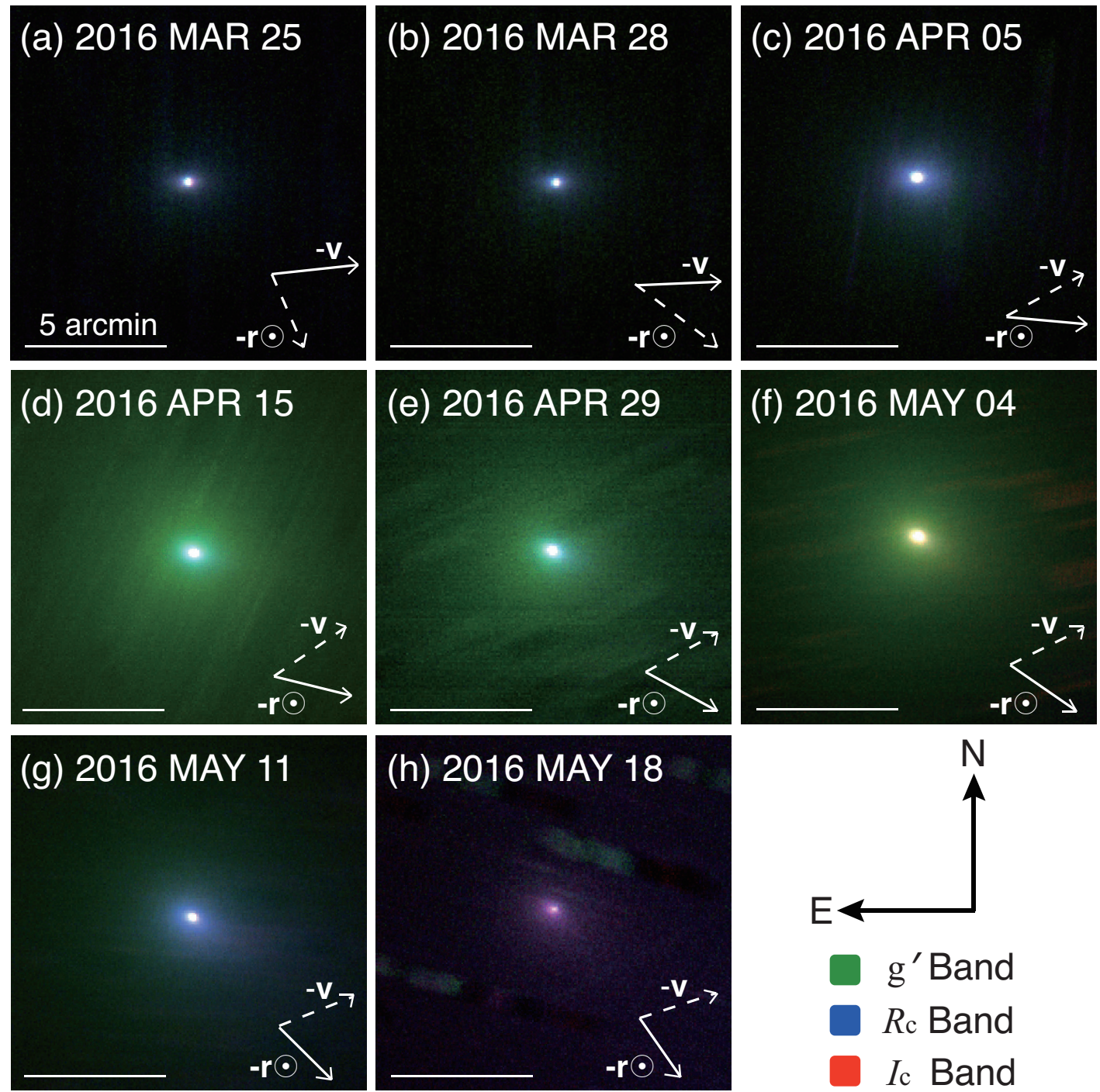

Fig. A.1. Wide-field composite images of 252P from the multiband OAO data. In each panel, the observation date and 5' scale bar are given at the top and bottom left, respectively. Denotations of arrows are identical with those in Fig. 2. North is up, and east is to the left. Green, blue, and red hues represent the fluxes in the $\mathrm{g}^{\prime}, R_{C}$, and $I_{C}$ bands, respectively. We note that the applied photometric aperture size of $1000 \mathrm{~km}$ in radius during this period decreased from $33.6^{\prime \prime}$ to $2.5^{\prime \prime}$, which is much smaller than the field of view of each panel.

\section{A.1. Photometric results}

Changes in the postperihelion activity level of $252 \mathrm{P}$ were quantified by the photometry, as in Sect. 3.1. The intrinsic brightness of 252P (Fig. 1a; derived from Eq. (7)) soon after perihelion passage seemed to decline steeply, but it began to rebound from the minimum of $\sim 17.3 \mathrm{mag}$ with a flux increase of nearly 16 times on UT 2016 March 27-28 (right arrow in Fig. 1a). Such re-ignition again became tranquil after peaking in approximately late April to early May at $\sim 14.3 \mathrm{mag}$. The changes in $A f \rho$ and $\dot{M}_{\mathrm{d}}$ patterns also showed similar trends with that of $m_{\mathrm{R}}(1,1,0)$, even though the first two have their maxima $\sim$ a week after the peak of $m_{\mathrm{R}}(1,1,0)$.

Over 1.5 months after the second activation, the $A f \rho$ values of $252 \mathrm{P}$ (Fig. 1b; derived from Eq. (8)) increased $\sim 57$ times from $14.1 \pm 3.8$ to $808.7 \pm 17.6 \mathrm{~cm}$. We compared the $A f \rho$ values with the HST data in the broadband F625W filter $\left(\lambda_{\mathrm{C}}=0.625 \mu \mathrm{m}\right)$ taken on UT 2016 March 14 and April $04(16.8 \pm 0.3 \mathrm{~cm}$ and
$57 \pm 1 \mathrm{~cm}$, respectively, from Li et al. 2017; cross points in Fig. 1b). There are consistent differences of $\sim 20 \mathrm{~cm}$ between the HST and OAO data observed at similar epochs, but we suspect that the different photometric aperture sizes employed (more than two orders of magnitude, i.e., $\rho \lesssim 10 \mathrm{~km}$ for the HST data and $\rho=1000 \mathrm{~km}$ for the OAO data) might cause the difference in $A f \rho$. Although, by definition, $A f \rho$ should be a constant value regardless of the aperture size under the steady expansion in the coma, such a condition would not be met in the vicinity of the nucleus. Considering that $A f \rho$ increases outward to reach an equilibrium (to $\sim 1000 \mathrm{~km}$ at $r_{\mathrm{H}} \sim 1 \mathrm{au}$; see, e.g., Bonev et al. 2008), it might be explainable that the OAO data in this study consistently showed higher Af $\rho$ than those of the HST data. Nonetheless, the HST data can be interpreted in line with the OAO data, in that $A f \rho$ of $252 \mathrm{P}$ in postperihelion was higher than that in preperihelion.

Similarly, variations in $\dot{M}_{\mathrm{d}}$ (Fig. 1c; derived from Eqs. (9) and (10)) strongly support the postperihelion reactivation of 


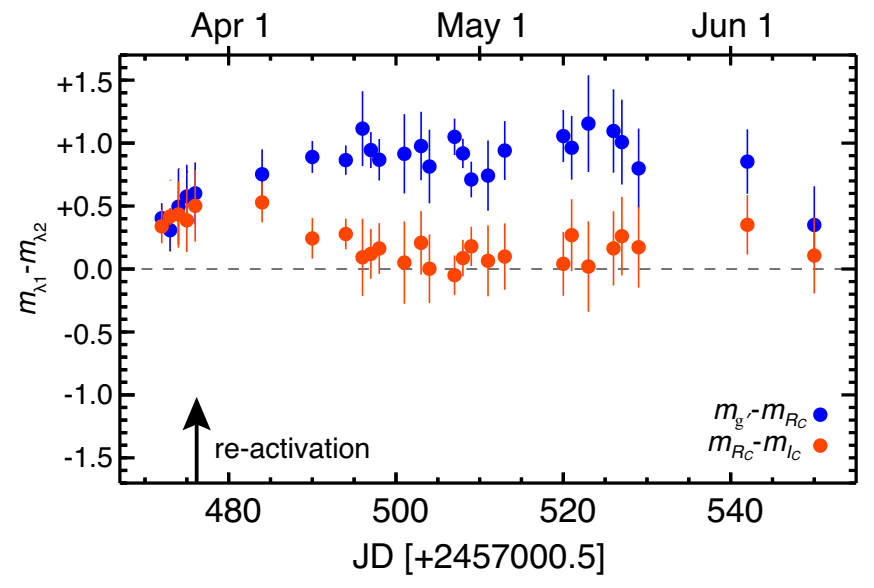

Fig. A.2. Temporal evolution in the postperihelion absolute magnitudes of $252 \mathrm{P}$ from the multiband $\mathrm{OAO}$ data. $m_{X}$ denotes the absolute magnitude in $X$ band, with an aperture size of $1000 \mathrm{~km}$ (corresponding to 33. $\left.6^{\prime \prime}-2.5^{\prime \prime}\right)$ in radius. Differences in $m_{X}$ between two bands are shown as blue $\left(m_{\mathrm{g}^{\prime}}-m_{R_{C}}\right)$ and orange $\left(m_{R_{C}}-m_{I_{C}}\right)$ circles. All the error bars include the $1 \sigma$ errors on the measurements, calibration of the field stars with the assumed internal catalog error of $\sim 0.1$, and propagated errors in magnitude subtraction of two bands.

252P, although the overall morphology of the evolutionary trend follows a concave shape, increasing and decreasing both in proportion to the third power of $r_{\mathrm{H}}$, unlike the convex shapes of the $m_{\mathrm{R}}(1,1,0)$ and $A f \rho$ trends. From the minimum of $1.1 \pm$ $1.0 \mathrm{~kg} \mathrm{~s}^{-1}$ on March 27 through the maximum of $31.3 \pm 2.1 \mathrm{~kg} \mathrm{~s}^{-1}$ on May 02 to another minimum of $4.5 \pm 2.4 \mathrm{~kg} \mathrm{~s}^{-1}$ on June 10 , the total mass-loss during the second activation event was $\sim 7.8 \times 10^{7} \mathrm{~kg}$, when we assumed nominal $\bar{a}=1 \mu \mathrm{m}$ dust grains. Taken as a whole, the derived parameters indicate that the second activation of 252P was likely on UT 2016 March 27-28.

\section{A.2. Variation in the coma color}

As a byproduct of our multiband imaging observation, we produced color images to clarify to what extent the fluxes from each band contributed to the postperihelion reactivation of the comet. The $\mathrm{g}^{\prime}, R_{C}$, and $I_{C}$ band images were allocated as green, blue, and red hues, respectively. Since we employed the broadband filters, gas contamination in flux estimation would be inevitable, particularly by the molecular emissions of the $\mathrm{C}_{2}$ Swan bands in the $\mathrm{g}^{\prime}$ filter, $\mathrm{NH}_{2} \alpha$ bands in the $R_{C}$ filter, and $\mathrm{CN}$ red bands in the $I_{C}$ filter (Meech \& Svoreň 2004).

Figure A.1 presents wide-field composite images, together with the dates of observation and $5^{\prime}$ scale bars in each panel, the fields of view of which are much larger than the applied photometric aperture size. These images show that greenish and bluish components spherically expanded from the nucleus once the comet became activated, whereas a whitish component elongated along the antisolar direction $\left(-\mathbf{r}_{\odot}\right)$. As the activity of the comet peaked, a greenish coma covered the entire field of view, developing a significant bluish green color in the innermost coma. The gas coma of $252 \mathrm{P}$ at this term spherically extended to $>10^{4} \mathrm{~km}$ from the nucleus in the sky plane (panels $\mathrm{d}$ to $\mathrm{f}$ ). Meanwhile, a reddish component was observably dominant, only after the reactivating event was completed (panel h).

Figure A.2 shows the temporal evolution of the $252 \mathrm{P}$ coma color in postperihelion more quantitatively. All reduced magnitudes were derived with the $1000 \mathrm{~km}$ aperture size (corresponding to $33.6^{\prime \prime}-2.5^{\prime \prime}$ ) in radius, the central most regions from the nucleus of each panel in Fig. A.1. The magnitude differences between the $\mathrm{g}^{\prime}-R_{C}$ and $R_{C}-I_{C}$ bands are given as blue $\left(m_{\mathrm{g}^{\prime}}-\right.$ $\left.m_{R_{C}}\right)$ and orange $\left(m_{R_{C}}-m_{I_{C}}\right)$ circles, respectively. The putative activation point (on March 27-28) is marked as an arrow. Overall, a blueward color change (i.e., predominant brightening in the $R_{C}$ band flux) is apparent in the inner coma. Before the activation, the comet was slightly red, showing on average $m_{\mathrm{g}^{\prime}}-m_{R_{C}} \sim$ 0.5 and $m_{R_{C}}-m_{I_{C}} \sim 0.4$ (almost identical within the error bars, however), which are physically indistinguishable from those of short-period comets in the inner solar system (Solontoi et al. 2012). During the activation, however, fluxes in the $R_{C}$ band became significantly enhanced, resulting in the redder color of $m_{\mathrm{g}^{\prime}}-m_{R_{C}}$ and more neutral color of $m_{R_{C}}-m_{I_{C}}$. These magnitude differences remained nearly constant at the value of $m_{\mathrm{g}^{\prime}}-$ $m_{R_{C}}=0.9 \pm 0.1$ (the standard deviation of the nominal values) and $m_{R_{C}}-m_{I_{C}}=0.2 \pm 0.1$ over the following 1.5 months. After peaking, the maximum $A f \rho$ and $\dot{M}_{\mathrm{d}}$, the coma color again turned to reddish-neutral, albeit with large uncertainties, consistent with the status before the activation.

\section{Appendix B: Orbital evolution of 252P/LINEAR and its dynamical association with P/2016 BA14 (PanSTARRS)}

In this section, we present our investigation of orbital evolution of 252P and its dynamical association with P/2016 BA14 (PanSTARRS) to search for any possible dynamical characteristics of the objects attributable to the observed photo-polarimetric properties of 252P.

Comet P/2016 BA14 (PanSTARRS; hereafter BA14), a possible dynamical pair of $252 \mathrm{P}$ with remarkably similar orbital elements (Rudawska et al. 2016), possesses a geometric albedo of $<3 \%$ (Reddy 2016) and diametric size of $>1 \mathrm{~km}$ (Naidu et al. 2016) or $1-2 \mathrm{~km}$ (Li et al. 2017). Unlike the capricious behavior of 252P, BA14 showed an $r^{\prime}$-band magnitude that was more than 6 mag larger with a fraction of active area on the surface of $\sim 0.01 \%$, (i.e., it was rather asteroidal in the 2016 apparition) (Li et al. 2017). To trace the dynamic history of the two back to the time when they were ejected into the near-Earth orbit, we conducted a backward dynamical simulation with the Mercury 6 integrator (Chambers 1999) under the gravity of the Sun and eight planets. We generated 1000 clones randomly distributed within the $1 \sigma$ Gaussian distribution, with the orbital elements quoted on the JPL Small-Body Database Browser site. They were integrated $1000 \mathrm{yr}$ backward with an 8-day time step in the general Bulirsch-Stoer mode without considering the Yarkovsky force (i.e., consistent with the negligible activity levels before the 2016 apparition). The applied orbital elements and their errors are summarized in Table 2.

In addition, to assess the orbital similarity of two bodies in the near-Earth orbit, we estimated a traditional $D_{\text {SH }}$ parameter (Southworth \& Hawkins 1963), which is a distance between the orbits of two bodies in five-dimensional orbital element space $(q, e, i, \omega$, and $\Omega)$ calculated as

$$
\begin{aligned}
D_{\mathrm{SH}}= & {\left[\left(e_{\mathrm{B}}-e_{\mathrm{A}}\right)^{2}+\left(q_{\mathrm{B}}-q_{\mathrm{A}}\right)^{2}\right.} \\
& \left.+\left(2 \sin \frac{I_{\mathrm{AB}}}{2}\right)^{2}+\left[\left(e_{\mathrm{A}}+e_{\mathrm{B}}\right) \sin \frac{\Pi_{\mathrm{AB}}}{2}\right]^{2}\right]^{1 / 2},
\end{aligned}
$$

where

$I_{\mathrm{AB}}=\arccos \left[\cos i_{\mathrm{A}} \cos i_{\mathrm{B}}+\sin i_{\mathrm{A}} \sin i_{\mathrm{B}} \cos \left(\Omega_{\mathrm{A}}-\Omega_{\mathrm{B}}\right)\right]$ 
Table B.1. Orbital elements of 252P and BA14 and their $1 \sigma$ uncertainties at Epoch 2457519.5 (=2016 May 11.0).

\begin{tabular}{ccccccc}
\hline \hline Object & $a^{(a)}$ & $e^{(b)}$ & $i^{(c)}$ & $\omega^{(d)}$ & $\Omega^{(e)}$ & $M^{(f)}$ \\
\hline \multirow{2}{*}{$252 \mathrm{P}$} & 3.0470 & 0.6731 & 10.4223 & 343.3110 & 190.9499 & 10.5137 \\
& $\left(6.3018 \times 10^{-5}\right)$ & $\left(6.8756 \times 10^{-6}\right)$ & $\left(6.3172 \times 10^{-5}\right)$ & $\left(5.4848 \times 10^{-5}\right)$ & $\left(6.2681 \times 10^{-5}\right)$ & $\left(3.000 \times 10^{-4}\right)$ \\
\hline \multirow{2}{*}{ BA14 } & 3.0216 & 0.6662 & 18.9181 & 351.9022 & 180.5312 & 40.8120 \\
& $\left(8.4014 \times 10^{-7}\right)$ & $\left(9.2653 \times 10^{-8}\right)$ & $\left(1.7085 \times 10^{-6}\right)$ & $\left(4.5491 \times 10^{-6}\right)$ & $\left(2.209 \times 10^{-6}\right)$ & $\left(1.7161 \times 10^{-5}\right)$ \\
\hline
\end{tabular}

Notes. ${ }^{(a)}$ The semimajor axis in au; ${ }^{(b)}$ the eccentricity; ${ }^{\left({ }^{c}\right)}$ the inclination in degrees; ${ }^{\left({ }^{d}\right)}$ the argument of perihelion in degrees; ${ }^{(e)}$ the longitude of the ascending node in degrees; and ${ }^{(f)}$ the mean anomaly in degrees. Numbers in parentheses are $1 \sigma$ uncertainties of the orbital elements. All the values are quoted from the JPL Small-Body Database Browser (https://ssd.jpl.nasa.gov/sbdb.cgi).

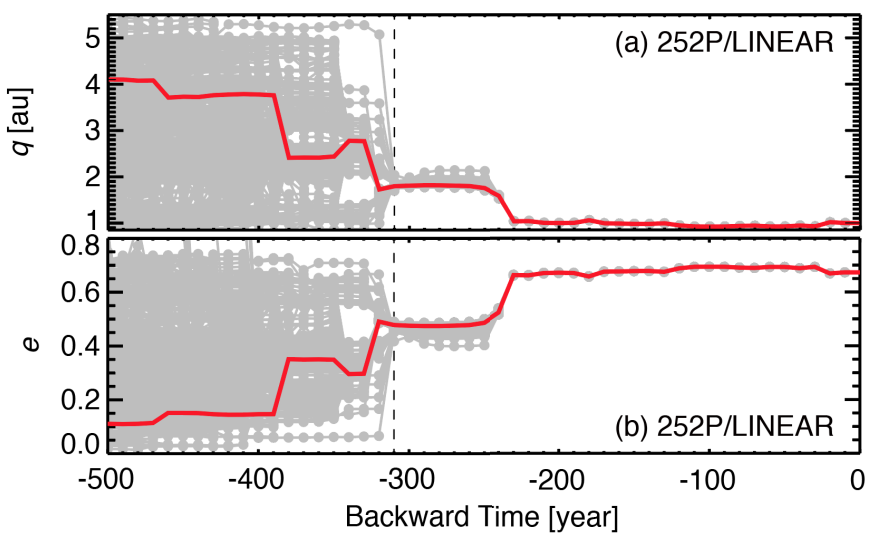

Fig. B.1. Time evolution of $(a)$ the perihelion distance $q$ and $(b)$ the eccentricity $e$ of 252P. Red lines denote the evolution of a particle with the average values at the epoch (May 11, 2016, the Julian date of 2457519.5 ), while each gray line represents that of an individual clone, the orbital elements of which follow $1 \sigma$ standard deviation of the Gaussian distribution.

and

$\Pi_{\mathrm{AB}}=\left(\omega_{\mathrm{A}}-\omega_{\mathrm{B}}\right)+2 \arcsin \left[\cos \frac{\left(i_{\mathrm{A}}+i_{\mathrm{B}}\right)}{2} \sin \frac{\left(\Omega_{\mathrm{A}}-\Omega_{\mathrm{B}}\right)}{2} \sec \frac{I_{\mathrm{AB}}}{2}\right]$.

The subscripts A and B denote the two bodies to be compared (A for 252P and B for BA14), $q$ is the perihelion distance in au, $e$ is the eccentricity, $i$ is the inclination, $\omega$ is the argument of perihelion, and $\Omega$ is the longitude of the ascending node. In the case of $D_{\mathrm{SH}} \leq 0.20$, we tend to consider that the two bodies are probably within the association range (Southworth \& Hawkins 1963). For instance, a minimum $D_{\mathrm{SH}}$ value of the possible pair, (3200) Phaethon and 2005 UD, shows 0.04 (Ohtsuka et al. 2006).

Figure B.1 shows the time evolution of the orbital elements of 252P. Red lines denote the evolution of a particle with the average values at the epoch (May 11, 2016, the Julian date of $2457519.5)$, while each gray line represents that of an individual clone, the orbital elements of which have $1 \sigma$ standard deviation on the Gaussian distribution. Our simulation shows that $252 \mathrm{P}$ would have closely encountered Jupiter at the minimum distance of $d_{\min } \sim 0.02 \mathrm{au}, 314 \mathrm{yr}$ ago. Due to the high uncertainty, we could only trace back the orbital evolution of the comet from the current epoch to $\sim 310 \mathrm{yr}$ in the past, during which the $q$ and $e$ of the comet changed by $\delta q \sim-1$ au (decreasing) and by $\delta e \sim+0.2$ (increasing). Interestingly, based on the current orbital elements of 252P, the Near-Earth Object (NEO) model of Greenstreet et al. (2012) suggests the two most probable source regions of the comet: the JFC source $(\sim 81.8 \%)$ and the outer main asteroidal belt $(\sim 16.2 \%)$. In either case, $252 \mathrm{P}$ should have eventually been transported to the current orbit by means of the resonance with Jupiter and the perturbations of terrestrial planets (e.g., Morbidelli et al. 2002). Based on the analysis, we conjecture that the decreasing $q$, together with its repetitive close encounters with planets (e.g., with Jupiter within $\sim 0.05$ au at $\sim 320 \mathrm{yr}$ ago and with the Earth within $\sim 0.02$ au since $\sim 170 \mathrm{yr}$ to the present), might introduce internal stresses to 252P, which would render the nucleus overall more fragile and would in turn affect the rapid polarimetric variation in its perihelion passage.

The average $D_{\mathrm{SH}}$ value derived from the osculating orbital elements of $252 \mathrm{P}$ and BA14 is $\sim 0.16$, which implies a positive dynamical correlation of two bodies, although not as tight as that of (3200) Phaethon and 2005 UD (Ohtsuka et al. 2006). Among all the possible combinations of the $1 \sigma$ clones, we obtained the minimum $D_{\mathrm{SH}}$ value of $\sim 0.15$. Owing to the close encounters of the objects to the planets and their nongravitational force, it is hard to determine from this study exactly when they were fragmented each other.

\section{Appendix C: Contrast in behaviors of large and small comet nuclei in the near-Earth orbit}

This section describes our conjecture of the reason why $252 \mathrm{P}$ and BA14 exhibited such different activity patterns during the 2016 apparition, despite their possible dynamical association resulting in similar orbital evolution in the near-Earth orbit.

The remarkably high activity of 252P in 2016 compared to the previous apparitions, alongside its discontinuous brightness enhancements near perihelion, is far different from the behavior of the comet over the past apparitions. We speculate that the effects of such orbital evolution on comets in nearEarth orbits might vary greatly depending on comet nuclei size. This speculation would be demonstrated by different observational characteristics of 252P and BA14 in the 2016 apparition, despite their similar orbital evolution over a few centuries (i.e., $D_{\mathrm{SH}} \leq 0.20$ in Appendix B). Comet 252P is on the small end of sizes $(300 \pm 30 \mathrm{~m}$; Li et al. 2017) of JFCs, for which a typical comet nucleus would range from one to a few kilometers in size (Fernández et al. 1999; Barnes et al. 2019). The surface gravity of $252 \mathrm{P}$ would then be less than a factor of at least one-fifth the gravity at the surface of ordinary JFCs. The concomitant smaller escape velocity permits large particles, millimeter to centimeter in size, to readily escape the gravitational influence of 252P, while a larger nucleus would more easily accumulate dust particles on its surface.

The small size can also contribute to the spin evolution of the nuclei such that the rotational stability tends to be proportional to the fourth power of the radius of the nucleus $\left(\tau_{\mathrm{ex}} \propto\right.$ $r_{\mathrm{n}}^{4}$; Jewitt 2004). Comet $252 \mathrm{P}$ thus has a $\gtrsim 10^{3}$ times smaller 
excitation timescale than that of a typical JFC. Indeed, a sudden increase of nongravitational force of $252 \mathrm{P}$ by a factor of $\sim 10$, but negligible change of BA14 during the 2016 apparition (Li et al. 2017) may reflect this higher rotational instability of the smaller comet.

Taken as a whole, the consequences of the small nucleus size together with the orbital evolution in the near-Earth orbit
(Appendix B) would enhance erratic sublimation patterns of the comet, probably resulting from highly changeable mass transfer on the surface (e.g., landslides; Keller et al. 2017) due to the enhanced solar radiation and/or in unstable development of the dust mantle on the surface (e.g., Rickman et al. 1990). They may in turn affect the abnormal variations of the photo-polarimetric parameters of 252P near its perihelion.

\section{Appendix D: Additional tables}

Table D.1. Observational geometry and instrument settings.

\begin{tabular}{|c|c|c|c|c|c|c|c|c|c|}
\hline Mode & Telescope/Instrument & $\begin{array}{l}\text { Median UT } \\
2016+\end{array}$ & Filter & $N$ & Exptime & $r_{\mathrm{H}}$ & $\Delta$ & $\alpha$ & $v$ \\
\hline \multirow{9}{*}{ Image } & \multirow{9}{*}{ LSGT/SBIG ST-10 } & 02/13 11:00 & \multirow{9}{*}{$R$} & 90 & 30.0 & 1.085 & 0.215 & 57.7 & 322.7 \\
\hline & & $02 / 2210: 53$ & & 100 & 30.0 & 1.042 & 0.168 & 67.1 & 332.8 \\
\hline & & $02 / 2410: 48$ & & 50 & 60.0 & 1.034 & 1.057 & 69.2 & 335.2 \\
\hline & & $02 / 2610: 38$ & & 50 & 60.0 & 1.027 & 0.146 & 71.3 & 337.6 \\
\hline & & 02/28 11:12 & & 25 & 60.0 & 1.021 & 0.135 & 73.4 & 340.0 \\
\hline & & 03/01 10:44 & & 50 & 60.0 & 1.015 & 0.125 & 75.4 & 342.5 \\
\hline & & 03/05 10:39 & & 100 & 30.0 & 1.006 & 0.103 & 79.4 & 347.5 \\
\hline & & 03/07 10:44 & & 65 & 30.0 & 1.002 & 0.092 & 81.3 & 350.0 \\
\hline & & 03/09 11:04 & & 100 & 5.0 & 0.999 & 0.081 & 83.1 & 352.6 \\
\hline \multirow{8}{*}{ Impol } & \multirow{6}{*}{ IRSF/SIRPOL } & 03/04 20:16 & \multirow{8}{*}{$J H K_{\mathrm{S}}$} & 60 & 20.0 & 1.007 & 0.106 & 78.8 & 346.7 \\
\hline & & 03/09 20:27 & & 60 & 10.0 & 0.999 & 0.079 & 83.5 & 353.1 \\
\hline & & 03/11 18:12 & & 20 & 5.0 & 0.997 & 0.069 & 85.0 & 355.5 \\
\hline & & 03/12 21:19 & & 100 & 5.0 & 0.997 & 0.063 & 85.8 & 356.9 \\
\hline & & $03 / 1319: 43$ & & 100 & 5.0 & 0.996 & 0.059 & 86.3 & 358.1 \\
\hline & & 03/14 18:49 & & 100 & 5.0 & 0.996 & 0.054 & 86.7 & 359.4 \\
\hline & \multirow[t]{2}{*}{ (Perihelion) } & $(03 / 1506: 22)$ & & $(\ldots)$ & $(\ldots)$ & $(0.996)$ & $(0.052)$ & $(86.8)$ & $(0.0)$ \\
\hline & & 03/15 19:56 & & 100 & 5.0 & 0.996 & 0.050 & 87.0 & 0.7 \\
\hline \multirow{27}{*}{ Image } & \multirow{27}{*}{ OAO-50/MITSUME } & 03/24 19:47 & \multirow{27}{*}{$\mathrm{g}^{\prime} R_{\mathrm{C}} I_{\mathrm{C}}$} & 12 & 30.0 & 1.005 & 0.041 & 77.6 & 12.2 \\
\hline & & $03 / 2519: 31$ & & 40 & 60.0 & 1.007 & 0.044 & 76.1 & 13.4 \\
\hline & & $03 / 2619: 20$ & & 50 & 60.0 & 1.009 & 0.048 & 74.8 & 14.7 \\
\hline & & $03 / 2718: 53$ & & 100 & 60.0 & 1.012 & 0.052 & 73.5 & 15.9 \\
\hline & & 03/28 18:55 & & 95 & 60.0 & 1.014 & 0.056 & 72.2 & 17.1 \\
\hline & & 04/05 19:30 & & 35 & 60.0 & 1.041 & 0.098 & 63.3 & 26.8 \\
\hline & & 04/11 18:09 & & 145 & 60.0 & 1.068 & 0.132 & 57.1 & 33.6 \\
\hline & & 04/15 17:24 & & 150 & 60.0 & 1.089 & 0.154 & 53.0 & 38.0 \\
\hline & & $04 / 17 \quad 17: 15$ & & 65 & 60.0 & 1.100 & 0.166 & 51.0 & 40.1 \\
\hline & & 04/18 17:44 & & 180 & 60.0 & 1.106 & 0.171 & 50.0 & 41.2 \\
\hline & & 04/19 16:49 & & 80 & 60.0 & 1.112 & 0.177 & 49.0 & 42.2 \\
\hline & & $04 / 22$ 17:02 & & 30 & 120.0 & 1.130 & 0.194 & 46.1 & 45.2 \\
\hline & & $04 / 24 \quad 17: 38$ & & 65 & 120.0 & 1.143 & 0.206 & 44.2 & 47.2 \\
\hline & & 04/25 16:59 & & 80 & 120.0 & 1.150 & 0.212 & 43.3 & 48.1 \\
\hline & & $04 / 28 \quad 16: 31$ & & 50 & 120.0 & 1.170 & 0.229 & 40.5 & 51.0 \\
\hline & & $04 / 29 \quad 17: 18$ & & 75 & 120.0 & 1.178 & 0.235 & 39.6 & 51.9 \\
\hline & & 04/30 17:14 & & 85 & 120.0 & 1.185 & 0.241 & 38.7 & 52.8 \\
\hline & & $05 / 0216: 56$ & & 30 & 120.0 & 1.199 & 0.253 & 37.0 & 54.6 \\
\hline & & 05/04 17:15 & & 60 & 120.0 & 1.214 & 0.266 & 35.2 & 56.4 \\
\hline & & $05 / 11 \quad 17: 15$ & & 70 & 120.0 & 1.268 & 0.310 & 29.8 & 62.1 \\
\hline & & $05 / 12$ 17:13 & & 70 & 120.0 & 1.276 & 0.317 & 29.1 & 62.9 \\
\hline & & 05/14 17:17 & & 45 & 120.0 & 1.292 & 0.331 & 27.7 & 64.5 \\
\hline & & 05/17 17:19 & & 20 & 120.0 & 1.316 & 0.352 & 25.8 & 66.7 \\
\hline & & 05/18 16:34 & & 80 & 120.0 & 1.324 & 0.359 & 25.2 & 67.4 \\
\hline & & $05 / 2016: 37$ & & 75 & 120.0 & 1.341 & 0.373 & 24.2 & 68.9 \\
\hline & & 06/02 18:00 & & 9 & 120.0 & 1.453 & 0.480 & 19.6 & 77.4 \\
\hline & & 06/10 17:58 & & 15 & 120.0 & 1.524 & 0.555 & 19.1 & 82.0 \\
\hline
\end{tabular}

Notes. Column headers: Mode, instrumental settings of imaging (Image) and imaging-polarimetry (Impol) observations; $N$, number of exposures; Exptime, individual exposure time in seconds; $r_{\mathrm{H}}$ and $\Delta$, median heliocentric and geocentric distances, respectively, in au; $\alpha$, median phase angle (angle of Sun-comet-observer) in degrees; and $v$, median true anomaly in degrees. We referred to the web-based JPL Horizons system (http://ssd.jpl.nasa.gov/?horizons) to obtain the ephemerides (as of 2019 Aug 28). 
Table D.2. Photometry of 252P/LINEAR.

\begin{tabular}{|c|c|c|c|c|c|}
\hline Telescope & $\begin{array}{c}\text { Median UT } \\
2016+\end{array}$ & $\varrho\left({ }^{\prime \prime}\right)$ & $m_{\mathrm{R}}(1,1,0)(\mathrm{mag})$ & $A f \rho(\mathrm{cm})$ & $\dot{M}_{\mathrm{d}}\left(\mathrm{kg} \mathrm{s}^{-1}\right)$ \\
\hline \multirow{9}{*}{ LSGT } & 02/13 11:00 & 6.41 & $18.67 \pm 0.30$ & $6.78 \pm 2.50$ & $0.38 \pm 0.14$ \\
\hline & $02 / 22 \quad 10: 53$ & 8.21 & $18.23 \pm 0.30$ & $7.51 \pm 2.50$ & $0.43 \pm 0.14$ \\
\hline & $02 / 2410: 48$ & 8.78 & $18.53 \pm 0.30$ & $5.33 \pm 2.45$ & $0.30 \pm 0.23$ \\
\hline & $02 / 2610: 38$ & 9.43 & $18.13 \pm 0.24$ & $7.23 \pm 2.20$ & $0.41 \pm 0.14$ \\
\hline & $02 / 2811: 12$ & 10.19 & $17.76 \pm 0.25$ & $9.48 \pm 2.25$ & $0.55 \pm 0.16$ \\
\hline & 03/01 10:44 & 11.07 & $17.78 \pm 0.25$ & $8.70 \pm 2.20$ & $0.50 \pm 0.15$ \\
\hline & 03/05 10:39 & 13.44 & $15.88 \pm 0.18$ & $44.14 \pm 1.48$ & $2.85 \pm 1.02$ \\
\hline & 03/07 10:44 & 15.03 & $15.81 \pm 0.14$ & $44.10 \pm 1.25$ & $2.82 \pm 1.00$ \\
\hline & 03/09 11:04 & 17.02 & $15.76 \pm 0.14$ & $43.56 \pm 1.24$ & $2.62 \pm 0.95$ \\
\hline \multirow{27}{*}{ OAO-50 } & $03 / 24$ 19:47 & 34.30 & $15.42 \pm 0.12$ & $71.05 \pm 3.24$ & $8.13 \pm 1.61$ \\
\hline & 03/25 19:31 & 31.96 & $16.64 \pm 0.13$ & $24.27 \pm 3.36$ & $3.38 \pm 1.65$ \\
\hline & $03 / 26 \quad 19: 20$ & 29.30 & $17.08 \pm 0.22$ & $16.94 \pm 4.25$ & $1.62 \pm 1.12$ \\
\hline & 03/27 18:53 & 27.05 & $17.33 \pm 0.19$ & $14.07 \pm 3.79$ & $1.08 \pm 0.97$ \\
\hline & $03 / 2818: 55$ & 25.11 & $16.90 \pm 0.25$ & $21.77 \pm 4.55$ & $1.43 \pm 1.27$ \\
\hline & 04/05 19:30 & 14.35 & $15.92 \pm 0.14$ & $71.04 \pm 3.40$ & $2.23 \pm 1.69$ \\
\hline & 04/11 18:09 & 10.65 & $14.89 \pm 0.13$ & $225.84 \pm 3.36$ & $3.94 \pm 1.65$ \\
\hline & 04/15 17:24 & 9.13 & $14.75 \pm 0.16$ & $291.78 \pm 5.15$ & $4.64 \pm 1.79$ \\
\hline & 04/17 17:15 & 8.47 & $15.27 \pm 0.26$ & $192.36 \pm 4.60$ & $3.08 \pm 2.30$ \\
\hline & 04/18 17:44 & 8.22 & $14.44 \pm 0.17$ & $427.23 \pm 8.00$ & $7.17 \pm 1.83$ \\
\hline & 04/19 16:49 & 7.95 & $14.55 \pm 0.17$ & $399.17 \pm 7.48$ & $6.95 \pm 1.86$ \\
\hline & 04/22 17:02 & 7.25 & $14.81 \pm 0.26$ & $346.12 \pm 8.48$ & $9.55 \pm 2.30$ \\
\hline & $04 / 24 \quad 17: 38$ & 6.83 & $14.27 \pm 0.21$ & $604.79 \pm 13.15$ & $21.04 \pm 2.07$ \\
\hline & $04 / 2516: 59$ & 6.63 & $14.57 \pm 0.23$ & $473.73 \pm 10.75$ & $18.63 \pm 2.14$ \\
\hline & $04 / 28 \quad 16: 31$ & 6.14 & $14.99 \pm 0.14$ & $351.14 \pm 5.62$ & $13.66 \pm 1.68$ \\
\hline & 04/29 17:18 & 5.98 & $14.79 \pm 0.12$ & $435.16 \pm 6.45$ & $16.94 \pm 1.60$ \\
\hline & 04/30 17:14 & 5.84 & $14.66 \pm 0.12$ & $504.63 \pm 7.54$ & $19.77 \pm 1.59$ \\
\hline & $05 / 02$ 16:56 & 5.56 & $14.26 \pm 0.22$ & $768.66 \pm 17.25$ & $31.25 \pm 2.09$ \\
\hline & $05 / 04$ 17:15 & 5.29 & $14.56 \pm 0.20$ & $614.42 \pm 12.53$ & $25.07 \pm 1.98$ \\
\hline & 05/11 17:15 & 4.54 & $14.54 \pm 0.19$ & $747.90 \pm 14.98$ & $26.37 \pm 1.93$ \\
\hline & $05 / 12 \quad 17: 13$ & 4.44 & $14.48 \pm 0.22$ & $808.69 \pm 17.57$ & $30.03 \pm 2.09$ \\
\hline & 05/14 17:17 & 4.25 & $14.86 \pm 0.33$ & $596.15 \pm 17.25$ & $21.62 \pm 2.66$ \\
\hline & 05/17 17:19 & 4.00 & $14.87 \pm 0.29$ & $628.16 \pm 16.44$ & $21.77 \pm 2.44$ \\
\hline & $05 / 18 \quad 16: 34$ & 3.92 & $14.99 \pm 0.30$ & $571.81 \pm 15.25$ & $20.33 \pm 2.54$ \\
\hline & 05/20 $16: 37$ & 3.77 & $15.04 \pm 0.29$ & $566.12 \pm 14.69$ & $20.24 \pm 2.44$ \\
\hline & 06/02 18:00 & 2.93 & $16.36 \pm 0.14$ & $194.27 \pm 3.37$ & $6.04 \pm 1.68$ \\
\hline & 06/10 17:58 & 2.53 & $16.55 \pm 0.27$ & $165.99 \pm 4.74$ & $4.46 \pm 2.35$ \\
\hline
\end{tabular}

Notes. All $\varrho$ values correspond to the projected physical distance of $1000 \mathrm{~km}$ from the nucleus center. 